\title{
Global existence of solutions and smoothing effects for classes of reaction-diffusion equations on manifolds
}

\author{
Gabriele Grilloid, Giulia Meglioli and Fabio Punzo
}

Abstract. We consider the porous medium equation with a power-like reaction term, posed on Riemannian manifolds. Under certain assumptions on $p$ and $m$ in (1.1), and for small enough nonnegative initial data, we prove existence of global in time solutions, provided that the Sobolev inequality holds on the manifold. Furthermore, when both the Sobolev and the Poincaré inequalities hold, similar results hold under weaker assumptions on the forcing term. By the same functional analytic methods, we investigate global existence for solutions to the porous medium equation with source term and variable density in $\mathbb{R}^{n}$.

\section{Introduction}

We investigate existence of global in time solutions to nonlinear reaction-diffusion problems of the following type:

$$
\left\{\begin{aligned}
u_{t} & =\Delta u^{m}+u^{p} & & \text { in } M \times(0, T) \\
u & =u_{0} & & \text { in } M \times\{0\},
\end{aligned}\right.
$$

where $M$ is an $N$-dimensional complete noncompact Riemannian manifold of infinite volume, $\Delta$ being the Laplace-Beltrami operator on $M$ and $T \in(0, \infty]$. We shall assume throughout this paper that

$$
N \geq 3, \quad m>1, \quad p>m,
$$

so that we are concerned with the case of degenerate diffusions of porous medium type (see [40]), and that the initial datum $u_{0}$ is nonnegative.

Let $\mathrm{L}^{q}(M)$ be the space of those measurable functions $f$ such that $|f|^{q}$ is integrable w.r.t. the Riemannian measure $\mu$. We shall always assume that $M$ supports the Sobolev inequality, namely that:

$$
\text { (Sobolev inequality) } \quad\|v\|_{L^{2^{*}(M)}} \leq \frac{1}{C_{S}}\|\nabla v\|_{L^{2}(M)} \text { for any } v \in C_{c}^{\infty}(M),
$$

Mathematics Subject Classification: Primary: 35K57, Secondary: 35B44, 58J35, 35K65, 35R01

Keywords: Reaction-diffusion equations, Riemannian manifolds, Blow-up, Global existence, Diffusions with weights. 
where $C_{s}$ is a positive constant and $2^{*}:=\frac{2 N}{N-2}$. In one of our main results, we shall also suppose that $M$ supports the Poincaré inequality, namely that:

$$
\text { (Poincaré inequality) } \quad\|v\|_{L^{2}(M)} \leq \frac{1}{C_{p}}\|\nabla v\|_{L^{2}(M)} \text { for any } v \in C_{c}^{\infty}(M),
$$

for some $C_{p}>0$. Observe that, for instance, (1.2) holds if $M$ is a Cartan-Hadamard manifold, i.e. a simply connected Riemannian manifold with nonpositive sectional curvatures, while (1.3) is valid when $M$ is a Cartan-Hadamard manifold satisfying the additional condition of having sectional curvatures bounded above by a constant $-c<0$ (see, e.g. [11,12]). Therefore, as is well known, in $\mathbb{R}^{N}$ (1.2) holds, but (1.3) fails, whereas on the hyperbolic space both (1.2) and (1.3) are fulfilled.

\subsection{On some existing results}

In [14], problem (1.1) has been studied when $p<m$. We refer the reader to such paper for a comprehensive account of the literature; here we limit ourselves to recall some results particularly related to ours.

For $M=\mathbb{R}^{N}$ and $m=1$, it is well known that, if $p \leq 1+\frac{2}{N}$, then the solution of problem (1.1) blows up in finite time for any $u_{0} \not \equiv 0$, while global existence holds if $p>1+\frac{2}{N}$ and $u_{0}$ is bounded and small enough (see [8,22]; for further results, see also $[7,9,10,25,32,35,36,39,44,45])$. For $m>1$, in [38] it is shown that the solution to problem (1.1) blows up for any $p \leq m+\frac{2}{N}, u_{0} \not \equiv 0$; instead, there exists a global in time solution provided $p>m+\frac{2}{N}$ and $u_{0}$ is compactly supported and sufficiently small. On Riemannian manifolds satisfying suitable volume growth conditions, for $m=1$ and $p \leq 1+\frac{2}{N}$, in $[29,46]$ it is proved that the solution of problem (1.1) blows up for any $u_{0} \not \equiv 0$, while global existence holds if $p>1+\frac{2}{N}$ for small enough initial data $u_{0}$. Similar results have also been stablished in $[5,34,42,43]$.

Problem (1.1), without the forcing term $u^{p}$, has been largely studied on Riemannian manifolds, and in particular on Cartan-Hadamard manifolds, in [6,13,15, 16, 18, 19 , $21,33,41]$. In [20] problem (1.1) is addressed on Cartan-Hadamard manifolds with $-k_{1} \leq$ sec $\leq-k_{2}$ for some $k_{1}>k_{2}>0$, where sec denotes the sectional curvature. It is shown that, for any $p>m$, there exists a global in time solution, provided that $u_{0}$ has compact support and is small enough, while if $u_{0}$ is large enough, then there exists a solution blowing up in finite time.

For any $x_{0} \in M, r>0$, let $B_{r}\left(x_{0}\right)$ be the geodesic ball centred in $x_{0}$ and radius $r$, let $g_{i j}$ the metric tensor. In [46], problem (1.1) is studied when $M$ is a manifold with a pole, $\mu\left(B_{r}\left(x_{0}\right)\right) \leq C r^{\alpha}$ for some $\alpha>2$ and $C>0$. Under an additional smallness condition on curvature at infinity, if $u_{0}$ is sufficiently small and with compact support, then there exists a global solution to problem (1.1). Global existence is also proved, for some initial data $u_{0}$, under the assumption that $M$ has nonnegative Ricci curvature and $p>\frac{\alpha}{\alpha-2} m$. It should be noticed that such result does not cover cases in which 
negative curvature either does not tend to zero at infinity, or does so not sufficiently fast, in particular the case of the hyperbolic space cannot be addressed.

Finally, in [14] global existence of solutions to problem (1.1) is obtained, for any $p<m$ and $u_{0} \in L^{m}(M)$, under the assumption that the Sobolev and the Poincare inequalities hold on $M$.

\subsection{Qualitative statements of our new results in the Riemannian setting}

Our results concerning problem (1.1) can be summarized as follows.

- (See Theorem 2.2) We prove global existence of solutions to (1.1), assuming that the initial datum is sufficiently small, that

$$
p>m+\frac{2}{N}
$$

and that the Sobolev inequality (1.2) holds; moreover, smoothing effects and the fact that suitable $L^{q}$ norms of solutions decrease in time are obtained. To be specific, any sufficiently small initial datum $u_{0} \in L^{m}(M) \cap L^{(p-m) \frac{N}{2}}(M)$ gives rise to a global solution $u(t)$ such that $u(t) \in L^{\infty}(M)$ for all $t>0$ with a quantitative bound on the $L^{\infty}$ norm of the solution.

- (See Theorem 2.5) We show that, if both the Sobolev and the Poincaré inequalities (i.e. (1.2), (1.3)) hold, then for any

$$
p>m
$$

for any sufficiently small initial datum $u_{0}$, belonging to suitable Lebesgue spaces, there exists a global solution $u(t)$ such that $u(t) \in L^{\infty}(M)$. Furthermore, a quantitative bound for the $L^{\infty}$ norm of the solution is satisfied for all $t>0$.

Note that in Theorem 2.2 we only assume the Sobolev inequality and we require that $p>m+\frac{2}{N}$, instead in Theorem 2.5 we can relax the assumption on the exponent $p$, indeed we assume $p>m$, but we need to further require that the Poincaré inequality holds. Moreover, in the two theorems, the hypotheses on the initial data are different.

The main results given in Theorems 2.2 and 2.5 depend essentially only on the validity of inequalities (1.2) and (1.3), are functional analytic in character and hence can be generalized to different contexts.

\subsection{The case of Euclidean, weighted diffusion}

As a particularly significant setting, we single out the case of Euclidean, massweighted reaction-diffusion equations, that has been the object of intense research. In fact, we consider the problem

$$
\begin{cases}\rho u_{t}=\Delta u^{m}+\rho u^{p} & \text { in } \mathbb{R}^{N} \times(0, T) \\ u=u_{0} & \text { in } \mathbb{R}^{N} \times\{0\},\end{cases}
$$


where $\rho: \mathbb{R}^{N} \rightarrow \mathbb{R}$ is strictly positive, continuous and bounded, and represents a mass variable density. The problem is naturally posed in the weighted spaces

$L_{\rho}^{q}\left(\mathbb{R}^{N}\right)=\left\{v: \mathbb{R}^{N} \rightarrow \mathbb{R}\right.$ measurable $\left.,\|v\|_{L_{\rho}^{q}}:=\left(\int_{\mathbb{R}^{N}} v^{q} \rho(x) d x\right)^{1 / q}<+\infty\right\}$.

This kind of problem arises in a physical model provided in [23]. Such choice of $\rho$ ensures that the following analogue of (1.2) holds:

$$
\|v\|_{L_{\rho}^{2 *}\left(\mathbb{R}^{N}\right)} \leq \frac{1}{C_{s}}\|\nabla v\|_{L^{2}\left(\mathbb{R}^{N}\right)} \text { for any } v \in C_{c}^{\infty}\left(\mathbb{R}^{N}\right)
$$

for a suitable positive constant $C_{S}$. In some cases, we also assume that the weighted Poincare inequality is valid, that is

$$
\|v\|_{L_{\rho}^{2}\left(\mathbb{R}^{N}\right)} \leq \frac{1}{C_{p}}\|\nabla v\|_{L^{2}\left(\mathbb{R}^{N}\right)} \text { for any } v \in C_{c}^{\infty}\left(\mathbb{R}^{N}\right)
$$

for some $C_{p}>0$. For example, (1.6) is fulfilled when $\rho(x) \asymp|x|^{-a}$, as $|x| \rightarrow+\infty$, for every $a \geq 2$, whereas, (1.5) is valid for every $a>0$.

Problem (1.4) under the assumption $1<p<m$ has been investigated in [14]. Under the assumption that the Poincare inequality is valid on $M$, it is shown that global existence and a smoothing effect for small $L^{m}$ initial data hold, that is solutions corresponding to such data are bounded for all positive times with a quantitative bound on their $L^{\infty}$ norm.

In [26,27], problem (1.4) is also investigated, under certain conditions on $\rho$. It is proved that if $\rho(x)=|x|^{-a}$ with $a \in(0,2)$,

$$
p>m+\frac{2-a}{N-a}
$$

and $u_{0} \geq 0$ is small enough, then a global solution exists (see [26, Theorem 1]). Note that the homogeneity of the weight $\rho(x)=|x|^{-a}$ is essentially used in the proof, since the Caffarelli-Kohn-Nirenberg estimate is exploited, which requires such a type of weight. In addition, a smoothing estimate holds. On the other hand, any nonnegative solution blows up, in a suitable sense, when $\rho(x)=|x|^{-a}$ or $\rho(x)=(1+|x|)^{-a}$ with $a \in[0,2), u_{0} \not \equiv 0$ and

$$
1<p<m+\frac{2-a}{N-a} .
$$

Furthermore, in [27,28], such results have been extended to more general initial data, decaying at infinity with a certain rate (see [27]). Finally, in [26, Theorem 2], it is shown that if $p>m, \rho(x)=(1+|x|)^{-a}$ with $a>2$, and $u_{0}$ is small enough, a global solution exists.

Problem (1.4) has also been studied in [30,31], by means of suitable barriers, supposing that the initial datum is continuous and with compact support. In particular, 
in [30] the case that $\rho(x) \asymp|x|^{-a}$ for $|x| \rightarrow+\infty$ with $a \in(0,2)$ is addressed. It is proved that for any $p>1$, if $u_{0}$ is large enough, then the solution blows up in finite time. On the other hand, if $p>\bar{p}$, for a certain $\bar{p}>m$ depending on $m, p$ and $\rho$, and $u_{0}$ is small enough, then there exists a global bounded solution. Moreover, in [31] the case that $a \geq 2$ is investigated. For $a=2$, blow-up is shown to occur when $u_{0}$ is big enough, whereas global existence holds when $u_{0}$ is small enough. For $a>2$, it is proved that if $p>m, u_{0} \in L_{\mathrm{loc}}^{\infty}\left(\mathbb{R}^{N}\right)$ and goes to 0 at infinity with a suitable rate, then there exists a global bounded solution. Furthermore, for the same initial datum $u_{0}$, if $1<p<m$, then there exists a global solution, which could blow up as $t \rightarrow+\infty$.

Our main results concerning problem (1.4) can be summarized as follows. Assume that $\rho \in C\left(\mathbb{R}^{N}\right) \cap L^{\infty}\left(\mathbb{R}^{N}\right), \rho>0$.

- (See Theorem 2.8) We prove that (1.4) admits a global solution, provided that

$$
p>m+\frac{2}{N}
$$

moreover, certain smoothing effects for solutions are fulfilled. More precisely, for any sufficiently small initial datum $u_{0} \in L_{\rho}^{m}\left(\mathbb{R}^{N}\right) \cap L_{\rho}^{(p-m) \frac{N}{2}}\left(\mathbb{R}^{N}\right)$ there exists a global solution $u(t)$ such that $u(t) \in L^{\infty}\left(\mathbb{R}^{N}\right)$ for all $t>0$ and a quantitative bound on the $L^{\infty}$ norm is verified. Moreover, suitable $L^{q}$ norms of solutions decrease in time.

- (See Theorem 2.9) We show that, if the Poincaré inequality (1.6) holds and one assumes the condition

$$
p>m
$$

then, for any sufficiently small initial datum $u_{0}$ belonging to suitable Lebesgue spaces, there exists a global solution $u(t)$ to (1.4) such that $u(t) \in L^{\infty}\left(\mathbb{R}^{N}\right)$, with a quantitative bound on the $L^{\infty}$ norm.

Let us compare our results with those in [26]. Theorem 2.8 deals with a different class of weights $\rho$ with respect to [26, Theorem 1], where $\rho(x)=|x|^{-a}$ for $a \in(0,2)$, and the homogeneity of $\rho$ is used. As a consequence, also the hypotheses on $p$ and the methods of proofs are different. Furthermore, Theorem 2.9 requires the validity of the Poincaré inequality, hence, in particular, it can be applied when $\rho(x)=(1+|x|)^{-a}$ with $a \geq 2$ (see [17]). On the other hand, in Theorem [26, Theorem 2] it is assumed that $\rho(x)=(1+|x|)^{-a}$ for $a>2$, so, the case $a=2$ is not included.

\subsection{Organization of the paper}

In Section 2, we state all our main results. In Section 3, some auxiliary results concerning elliptic problems are deduced together with a Benilan-Crandall-type estimate. In Section 4, we introduce a family of approximating problems. Then, for such solutions, we prove that suitable $L^{q}$ norms of solutions decrease in time, and a smoothing estimate, in the case $p>m+\frac{2}{N}$, supposing that $M$ supports the Sobolev 
inequality. Under such assumptions, global existence for problem (1.1) is shown in Section 5. In Section 6, we prove that suitable $L^{q}$ norms of solutions decrease in time, and $L^{\infty}$ bounds for solutions of the approximating problems, under the assumptions that $p>m$ and that $M$ supports the Poincaré inequality as well. Then, under such hypotheses, existence of global solutions to problem (1.1) is proved. Finally, a concise proof of the results concerning problem (1.4) is given in Section 7 by adapting the previous methods to that situation.

\section{Statements of main results}

We state first our results concerning solutions to problem (1.1), then we pass to the ones valid for solutions to problem (1.4).

\subsection{Global existence on Riemannian manifolds}

Solutions to (1.1) will be meant in the very weak, or distributional, sense, according to the following definition.

Definition 2.1. Let $M$ be a complete noncompact Riemannian manifold of infinite volume. Let $m>1, p>m$ and $u_{0} \in \mathrm{L}_{l o c}^{1}(M), u_{0} \geq 0$. We say that the function $u$ is a solution to problem (1.1) in the time interval $[0, T)$ if

$$
u \in L_{l o c}^{p}(M \times(0, T))
$$

and for any $\varphi \in C_{c}^{\infty}(M \times[0, T])$ such that $\varphi(x, T)=0$ for any $x \in M, u$ satisfies the equality:

$$
\begin{aligned}
-\int_{0}^{T} \int_{M} u \varphi_{t} d \mu d t= & \int_{0}^{T} \int_{M} u^{m} \Delta \varphi d \mu d t+\int_{0}^{T} \int_{M} u^{p} \varphi d \mu d t \\
& +\int_{M} u_{0}(x) \varphi(x, 0) d \mu .
\end{aligned}
$$

First, we consider the case that $p>m+\frac{2}{N}$ and the Sobolev inequality holds on $M$. In order to state our results, we define

$$
p_{0}:=(p-m) \frac{N}{2} .
$$

Observe that $p_{0}>1$ whenever $p>m+\frac{2}{N}$.

Theorem 2.2. Let $M$ be a complete, noncompact manifold of infinite volume such that the Sobolev inequality (1.2) holds. Let $m>1, p>m+\frac{2}{N}$ and $u_{0} \in L^{m}(M) \cap L^{p_{0}}(M)$, $u_{0} \geq 0$ where $p_{0}$ has been defined in (2.1). Let

$$
r>\max \left\{p_{0}, \frac{N}{2}\right\}, \quad s=1+\frac{2}{N}-\frac{1}{r} .
$$


Assume that

$$
\left\|u_{0}\right\|_{L^{p_{0}}(M)}<\varepsilon_{0}
$$

with $\varepsilon_{0}=\varepsilon_{0}\left(p, m, N, r, C_{s}\right)$ sufficiently small. Then, problem (1.1) admits a solution for any $T>0$, in the sense of Definition 2.1. Moreover, for any $\tau>0$, one has $u \in L^{\infty}(M \times(\tau,+\infty))$ and there exists a numerical constant $\Gamma>0$ such that, for all $t>0$, one has

$$
\|u(t)\|_{L^{\infty}(M)} \leq \Gamma t^{-\frac{\gamma}{m s}}\left\{\left\|u_{0}\right\|_{L^{p_{0}(M)}}^{\delta_{1}}+\left\|u_{0}\right\|_{L^{p_{0}(M)}}^{\delta_{2}}\right\}^{\frac{1}{m s}}\left\|u_{0}\right\|_{L^{m}(M)}^{\frac{s-1}{s}},
$$

where

$$
\begin{aligned}
& \gamma=\frac{p}{p-1}\left[1-\frac{N(p-m)}{2 p r}\right], \quad \delta_{1}=p \frac{p-m}{p-1}\left[1+\frac{N(m-1)}{2 p r}\right], \\
& \delta_{2}=\frac{p-m}{p-1}\left[1+\frac{N(m-1)}{2 r}\right] .
\end{aligned}
$$

Moreover, let $p_{0} \leq q<\infty$ and

$$
\left\|u_{0}\right\|_{L^{p_{0}(M)}}<\hat{\varepsilon}_{0}
$$

for $\hat{\varepsilon}_{0}=\hat{\varepsilon}_{0}\left(p, m, N, r, C_{s}, q\right)$ small enough. Then, there exists a constant $C=$ $C\left(m, p, N, \varepsilon_{0}, C_{s}, q\right)>0$ such that

$$
\|u(t)\|_{L^{q}(M)} \leq C t^{-\gamma_{q}}\left\|u_{0}\right\|_{L^{p_{0}(M)}}^{\delta_{q}} \text { for all } t>0,
$$

where

$$
\gamma_{q}=\frac{1}{p-1}\left[1-\frac{N(p-m)}{2 q}\right], \quad \delta_{q}=\frac{p-m}{p-1}\left[1+\frac{N(m-1)}{2 q}\right] .
$$

Finally, for any $1<q<\infty$, if $u_{0} \in L^{q}(M) \cap L^{p_{0}}(M) \cap L^{m}(M)$ and

$$
\left\|u_{0}\right\|_{L^{p_{0}}(M)}<\varepsilon
$$

with $\varepsilon=\varepsilon\left(p, m, N, r, C_{s}, q\right)$ sufficiently small, then

$$
\|u(t)\|_{L^{q}(M)} \leq\left\|u_{0}\right\|_{L^{q}(M)} \text { for all } t>0 .
$$

Remark 2.3. We notice that the proof of the above theorem will show that one can take an explicit value of $\varepsilon_{0}$ in (2.2). In fact, let $q_{0}>1$ be fixed and $\left\{q_{n}\right\}_{n \in \mathbb{N}}$ be the sequence defined by:

$$
q_{n}=\frac{N}{N-2}\left(m+q_{n-1}-1\right), \quad \forall n \in \mathbb{N},
$$

so that

$$
q_{n}=\left(\frac{N}{N-2}\right)^{n} q_{0}+\frac{N(m-1)}{N-2} \sum_{i=0}^{n-1}\left(\frac{N}{N-2}\right)^{i} .
$$


Clearly, $\left\{q_{n}\right\}$ is increasing and $q_{n} \longrightarrow+\infty$ as $n \rightarrow+\infty$. Fix $q \in\left[q_{0},+\infty\right)$ and let $\bar{n}$ be the first index such that $q_{\bar{n}} \geq q$. Define

$$
\begin{aligned}
\tilde{\varepsilon}_{0} & =\tilde{\varepsilon}_{0}\left(p, m, N, C_{s}, q, q_{0}\right) \\
& :=\left[\min \left\{\min _{n=0, \ldots, \bar{n}} \frac{2 m\left(q_{n}-1\right)}{\left(m+q_{n}-1\right)^{2}} C_{s}^{2} ; \frac{2 m\left(p_{0}-1\right)}{\left(m+p_{0}-1\right)^{2}} C_{s}^{2}\right\}\right]^{\frac{1}{p-m}} .
\end{aligned}
$$

Observe that $\varepsilon_{0}$ in (2.8) depends on the value of $q$ through the sequence $\left\{q_{n}\right\}$. More precisely, $\bar{n}$ is increasing with respect to $q$, while the quantity $\min _{n=0, \ldots, \bar{n}} \frac{2 m\left(q_{n}-1\right)}{\left(m+q_{n}-1\right)^{2}} C_{s}^{2}$ decreases w.r.t. $q$. We then let $q_{0}=p_{0}$, take $q=p r$ and define, for these choice of $q_{0}, q$,

$$
\varepsilon_{0}=\varepsilon_{0}\left(p, m, N, C_{s}, r\right)=\tilde{\varepsilon}_{0}\left(p, m, N, C_{s}, p r, p_{0}\right) .
$$

Furthermore, in (2.3) we can take

$$
\hat{\varepsilon}_{0}=\hat{\varepsilon}_{0}\left(p, m, N, C_{s}, q\right)=\tilde{\varepsilon}_{0}\left(p, m, N, C_{s}, q, p_{0}\right) .
$$

Similarly, one can choose the following explicit value for $\varepsilon$ in (2.5):

$$
\varepsilon=\bar{\varepsilon} \wedge \varepsilon_{0},
$$

where

$$
\bar{\varepsilon}=\bar{\varepsilon}\left(p, m, C_{s}, q\right):=\left[\min \left\{\frac{2 m(q-1)}{(m+q-1)^{2}} C_{s}^{2} ; \frac{2 m\left(p_{0}-1\right)}{\left(m+p_{0}-1\right)^{2}} C_{s}^{2}\right\}\right]^{\frac{1}{p-m}} .
$$

Remark 2.4. Observe that, for $M=\mathbb{R}^{N}$, in [38, Theorem 3, p. 220] it is shown that if $p>m+\frac{2}{N}$ and $u_{0}$ has compact support and is small enough, then the solution to problem (1.1) globally exists and decays like

$$
t^{-\frac{1}{p-1}} \quad \text { as } t \longrightarrow+\infty \text {. }
$$

Note that under these assumptions, Theorem 2.2 can be applied. It implies that the solution to problem (1.1) globally exists and decays like

$$
t^{-\frac{\gamma}{m s}} \quad \text { as } \quad t \longrightarrow+\infty \text {. }
$$

It is easily seen that, for any $p \geq m\left(1+\frac{2}{N}\right)$,

$$
\frac{\gamma}{m s} \geq \frac{1}{p-1}
$$

instead, for any $m+\frac{2}{N}<p<m\left(1+\frac{2}{N}\right)$,

$$
\frac{\gamma}{m s}<\frac{1}{p-1} \text {. }
$$

Hence, when $p \geq m\left(1+\frac{2}{N}\right)$ the decay's rate of the solution $u(t)$, for large times, given by Theorem 2.2 is better than that of [38, Theorem 3, p. 220], while the opposite is true for $m+\frac{2}{N}<p<m\left(1+\frac{2}{N}\right)$. In both cases, the class of initial data considered in Theorem 2.2 is wider. 
In the next theorem, we address the case that $p>m$, supposing that both the inequalities (1.2) and (1.3) hold on $M$.

Theorem 2.5. Let $M$ be a complete, noncompact manifold of infinite volume such that the Sobolev inequality (1.2) and the Poincaré inequality (1.3) hold. Let

$$
m>1, \quad p>m, \quad r>\frac{N}{2},
$$

and $u_{0} \in L^{\theta}(M) \cap L^{p r}(M)$ where $\theta=\min \{m, r\}, u_{0} \geq 0$. Let

$$
s=1+\frac{2}{N}-\frac{1}{r} \text {. }
$$

Assume that

$$
\left\|u_{0}\right\|_{L^{p \frac{N}{2}}(M)}<\varepsilon_{1}
$$

holds with $\varepsilon_{1}=\varepsilon_{1}\left(m, p, N, r, C_{p}, C_{s}\right)$ sufficiently small. Then, problem (1.1) admits a solution for any $T>0$, in the sense of Definition 2.1. Moreover, for any $\tau>0$ one has $u \in L^{\infty}(M \times(\tau,+\infty))$ and for all $t>0$ one has

$$
\|u(t)\|_{L^{\infty}(M)} \leq\left(\frac{s}{s-1}\right)^{\frac{1}{m}}\left\|u_{0}\right\|_{L^{m}(M)}^{\frac{s-1}{s}}\left[\left\|u_{0}\right\|_{L^{p r}(M)}^{p}+\frac{1}{(m-1) t}\left\|u_{0}\right\|_{L^{r}(M)}\right]^{\frac{1}{m s}}
$$

Moreover, suppose that $u_{0} \in L^{q}(M) \cap L^{\theta}(M) \cap L^{p r}(M)$ for some for $1<q<\infty$,

$$
\left\|u_{0}\right\|_{L^{p \frac{N}{2}}(M)}<\varepsilon_{2},
$$

for some $\varepsilon_{2}=\varepsilon_{2}\left(p, m, N, r, C_{p}, C_{s}, q\right)$ sufficiently small. Then,

$$
\|u(t)\|_{L^{q}(M)} \leq\left\|u_{0}\right\|_{L^{q}(M)} \text { for all } t>0 \text {. }
$$

Remark 2.6. We define, given $q>1$ :

$$
\tilde{\varepsilon}_{1}(q):=\left[\min \left\{\frac{2 m(q-1)}{(m+q-1)^{2}} C ; \frac{2 m\left(p \frac{N}{2}-1\right)}{\left(m+p \frac{N}{2}-1\right)^{2}} C\right\}\right]^{\frac{p+m+q-1}{p(p+q-1)-m(m+q-1)}}
$$

where $C=C_{p}^{2 m / p} \tilde{C}$ and $\tilde{C}=\tilde{C}\left(C_{s}, m, p, q\right)>0$ is defined in (6.8), with the choice $\theta:=\frac{m(m+q-1)}{p(p+q-1)}$. The proof will show that one can choose $\varepsilon_{1}:=\min _{i=1, \ldots, 4} \tilde{\varepsilon}_{1}\left(q_{i}\right)$ where $q_{1}=m, q_{2}=p, q_{3}=p r$ and $q_{4}=r$.

Similarly, we observe that in (2.12) we can choose

$$
\varepsilon_{2}=\varepsilon_{1} \wedge \tilde{\varepsilon}_{1}(q)
$$

In the next sections, we always keep the notation as in Remarks 2.3 and 2.6. 
2.2. Weighted, Euclidean reaction-diffusion problems

We consider a weight $\rho: \mathbb{R}^{N} \rightarrow \mathbb{R}$ such that

$$
\rho \in C\left(\mathbb{R}^{N}\right) \cap L^{\infty}\left(\mathbb{R}^{N}\right), \quad \rho(x)>0 \text { for any } x \in \mathbb{R}^{N} .
$$

Solutions to problem (1.4) are meant according to the following definition.

Definition 2.7. Let $m>1, p>m$ and $u_{0} \in \mathrm{L}_{\rho, l o c}^{1}\left(\mathbb{R}^{N}\right), u_{0} \geq 0$. Let the weight $\rho$ satisfy (2.16). We say that the function $u$ is a solution to problem (1.4) in the interval $[0, T)$ if

$$
u \in L_{\rho, l o c}^{p}\left(\mathbb{R}^{N} \times(0, T)\right)
$$

and for any $\varphi \in C_{c}^{\infty}\left(\mathbb{R}^{N} \times[0, T]\right)$ such that $\varphi(x, T)=0$ for any $x \in \mathbb{R}^{N}, u$ satisfies the equality:

$$
\begin{aligned}
-\int_{0}^{T} \int_{\mathbb{R}^{N}} u \varphi_{t} \rho(x) d x d t= & \int_{0}^{T} \int_{\mathbb{R}^{N}} u^{m} \Delta \varphi d x d t+\int_{0}^{T} \int_{\mathbb{R}^{N}} u^{p} \varphi \rho(x) d x d t \\
& +\int_{\mathbb{R}^{N}} u_{0}(x) \varphi(x, 0) \rho(x) d x
\end{aligned}
$$

First, we consider the case that $p>m+\frac{2}{N}$. Recall that since $\rho$ is bounded, the Sobolev inequality (1.5) necessarily holds.

Theorem 2.8. Let $\rho$ satisfy (2.16). Let $m>1, p>m+\frac{2}{N}$ and $u_{0} \in L_{\rho}^{m}\left(\mathbb{R}^{N}\right) \cap$ $L_{\rho}^{p_{0}}\left(\mathbb{R}^{N}\right), u_{0} \geq 0$ with $p_{0}$ defined in (2.1). Let

$$
r>\max \left\{p_{0}, \frac{N}{2}\right\}, \quad s=1+\frac{2}{N}-\frac{1}{r} .
$$

Assume that

$$
\left\|u_{0}\right\|_{L_{\rho}^{p_{0}}\left(\mathbb{R}^{N}\right)}<\varepsilon_{0}
$$

with $\varepsilon_{0}=\varepsilon_{0}\left(p, m, N, r, C_{s}\right)$ sufficiently small. Then, problem (1.4) admits a solution for any $T>0$, in the sense of Definition 2.7. Moreover, for any $\tau>0$, one has $u \in L^{\infty}\left(\mathbb{R}^{N} \times(\tau,+\infty)\right)$ and there exist $\Gamma>0$ such that, for all $t>0$, one has

$$
\|u(t)\|_{L^{\infty}\left(\mathbb{R}^{N}\right)} \leq \Gamma t^{-\frac{\gamma}{m s}}\left\{\left\|u_{0}\right\|_{L_{\rho}^{p_{0}}\left(\mathbb{R}^{N}\right)}^{\delta_{1}}+\frac{1}{m-1}\left\|u_{0}\right\|_{L_{\rho}^{p_{0}}\left(\mathbb{R}^{N}\right)}^{\delta_{2}}\right\}^{\frac{1}{m s}}\left\|u_{0}\right\|_{L_{\rho}^{m}\left(\mathbb{R}^{N}\right)}^{\frac{s-1}{s}},
$$

where

$$
\begin{aligned}
& \gamma=\frac{p}{p-1}\left[1-\frac{N(p-m)}{2 p r}\right], \delta_{1}=p \frac{p-m}{p-1}\left[1+\frac{N(m-1)}{2 p r}\right], \\
& \delta_{2}=\frac{p-m}{p-1}\left[1+\frac{N(m-1)}{2 r}\right] .
\end{aligned}
$$


Moreover, let $p_{0} \leq q<\infty$ and

$$
\left\|u_{0}\right\|_{L_{\rho}^{p_{0}}\left(\mathbb{R}^{N}\right)}<\hat{\varepsilon}_{0}
$$

for $\hat{\varepsilon}_{0}=\hat{\varepsilon}_{0}\left(p, m, N, r, C_{s}, q\right)$ small enough. Then, there exists a constant $C=$ $C\left(m, p, N, \varepsilon_{0}, C_{s}, q\right)>0$ such that

$$
\|u(t)\|_{L_{\rho}^{q}\left(\mathbb{R}^{N}\right)} \leq C t^{-\gamma_{q}}\left\|u_{0}\right\|_{L_{\rho}^{p_{0}}\left(\mathbb{R}^{N}\right)}^{\delta_{q}} \text { for all } t>0
$$

where

$$
\gamma_{q}=\frac{1}{p-1}\left[1-\frac{N(p-m)}{2 q}\right], \quad \delta_{q}=\frac{p-m}{p-1}\left[1+\frac{N(m-1)}{2 q}\right] .
$$

Finally, for any $1<q<\infty$, if $u_{0} \in L_{\rho}^{q}\left(\mathbb{R}^{N}\right) \cap L_{\rho}^{p_{0}}\left(\mathbb{R}^{N}\right) \cap L_{\rho}^{m}\left(\mathbb{R}^{N}\right)$ and

$$
\left\|u_{0}\right\|_{L_{\rho}^{p_{0}}\left(\mathbb{R}^{N}\right)}<\varepsilon
$$

holds, with $\varepsilon=\varepsilon\left(p, m, N, r, C_{s}, q\right)$ sufficiently small, then

$$
\|u(t)\|_{L_{\rho}^{q}\left(\mathbb{R}^{N}\right)} \leq\left\|u_{0}\right\|_{L_{\rho}^{q}\left(\mathbb{R}^{N}\right)} \text { for all } t>0 .
$$

A quantitative form of the smallness condition on $u_{0}$ in the above theorem can be given exactly as in Remark 2.3, see in particular (2.8), (2.9) and (2.10).

In the next theorem, we address the case $p>m$. We suppose that the Poincaré inequality (1.6) holds.

Theorem 2.9. Let $\rho$ satisfy (2.16) and assume that the inequality (1.6) hold. Let

$$
m>1, \quad p>m, \quad r>\frac{N}{2},
$$

and $u_{0} \in L_{\rho}^{\theta}\left(\mathbb{R}^{N}\right) \cap L_{\rho}^{p r}\left(\mathbb{R}^{N}\right)$ where $\theta=\min \{m, r\}, u_{0} \geq 0$. Let

$$
s=1+\frac{2}{N}-\frac{1}{r}
$$

Assume that

$$
\left\|u_{0}\right\|_{L_{\rho}^{p \frac{N}{2}}\left(\mathbb{R}^{N}\right)}<\varepsilon_{1}
$$

holds with $\varepsilon_{1}=\varepsilon_{1}\left(m, p, N, r, C_{p}, C_{s}\right)$ sufficiently small. Then, problem (1.4) admits a solution for any $T>0$, in the sense of Definition 2.7. Moreover, for any $\tau>0$ one has $u \in L^{\infty}\left(\mathbb{R}^{N} \times(\tau,+\infty)\right)$ and for all $t>0$ one has

$$
\|u(t)\|_{L^{\infty}\left(\mathbb{R}^{N}\right)} \leq\left(\frac{s}{s-1}\right)^{\frac{1}{m}}\left\|u_{0}\right\|_{L_{\rho}^{m}\left(\mathbb{R}^{N}\right)}^{\frac{s-1}{s}}\left[\left\|u_{0}\right\|_{L_{\rho}^{p r}\left(\mathbb{R}^{N}\right)}^{p}+\frac{1}{(m-1) t}\left\|u_{0}\right\|_{L_{\rho}^{r}\left(\mathbb{R}^{N}\right)}\right]^{\frac{1}{m s}} .
$$


Moreover, suppose that $u_{0} \in L_{\rho}^{q}\left(\mathbb{R}^{N}\right) \cap L_{\rho}^{\theta}\left(\mathbb{R}^{N}\right) \cap L_{\rho}^{p r}\left(\mathbb{R}^{N}\right)$ for some for $1<q<\infty$,

$$
\left\|u_{0}\right\|_{L_{\rho}^{p \frac{N}{2}}\left(\mathbb{R}^{N}\right)}<\varepsilon_{2},
$$

for some $\varepsilon_{2}=\varepsilon_{2}\left(p, m, N, r, C_{p}, C_{s}, q\right)$ small enough. Then,

$$
\|u(t)\|_{L_{\rho}^{q}\left(\mathbb{R}^{N}\right)} \leq\left\|u_{0}\right\|_{L_{\rho}^{q}\left(\mathbb{R}^{N}\right)} \text { for all } t>0 .
$$

A quantitative form of the smallness condition on $u_{0}$ in the above theorem can be given exactly as in Remark 2.6, see in particular (2.14) and (2.15).

\section{Auxiliary results for elliptic problems}

Let $x_{0}, x \in M$. We denote by $r(x)=\operatorname{dist}\left(x_{0}, x\right)$ the Riemannian distance between $x_{0}$ and $x$. Moreover, we let $B_{R}\left(x_{0}\right):=\left\{x \in M\right.$, dist $\left.\left(x_{0}, x\right)<R\right\}$ be the geodesic ball with centre $x_{0} \in M$ and radius $R>0$. If a reference point $x_{0} \in M$ is fixed, we shall simply denote by $B_{R}$ the ball with centre $x_{0}$ and radius $R$. Moreover, we denote by $\mu$ the Riemannian measure on $M$.

For any given function $v$, we define for any $k \in \mathbb{R}^{+}$

$$
T_{k}(v):= \begin{cases}k & \text { if } v \geq k \\ v & \text { if }|v|<k \\ -k & \text { if } v \leq-k\end{cases}
$$

For every $R>0, k>0$, consider the problem

$$
\begin{cases}u_{t}=\Delta u^{m}+T_{k}\left(u^{p}\right) & \text { in } B_{R} \times(0,+\infty) \\ u=0 & \text { in } \partial B_{R} \times(0,+\infty) \\ u=u_{0} & \text { in } B_{R} \times\{0\},\end{cases}
$$

where $u_{0} \in L^{\infty}\left(B_{R}\right), u_{0} \geq 0$. Solutions to problem (3.2) are meant in the weak sense as follows.

Definition 3.1. Let $m>1$ and $p>m$. Let $u_{0} \in L^{\infty}\left(B_{R}\right), u_{0} \geq 0$. We say that a nonnegative function $u$ is a solution to problem (3.2) if

$$
u \in L^{\infty}\left(B_{R} \times(0,+\infty)\right), u^{m} \in L^{2}\left((0, T) ; H_{0}^{1}\left(B_{R}\right)\right) \quad \text { for any } T>0,
$$

and for any $T>0, \varphi \in C_{c}^{\infty}\left(B_{R} \times[0, T]\right)$ such that $\varphi(x, T)=0$ for every $x \in B_{R}$, $u$ satisfies the equality:

$$
\begin{aligned}
-\int_{0}^{T} \int_{B_{R}} u \varphi_{t} d \mu d t= & -\int_{0}^{T} \int_{B_{R}}\left\langle\nabla u^{m}, \nabla \varphi\right\rangle d \mu d t+\int_{0}^{T} \int_{B_{R}} T_{k}\left(u^{p}\right) \varphi d \mu d t \\
& +\int_{B_{R}} u_{0}(x) \varphi(x, 0) d \mu .
\end{aligned}
$$


We also consider elliptic problems of the type

$$
\left\{\begin{aligned}
-\Delta u=f & \text { in } \quad B_{R} \\
u=0 & \text { in } \quad \partial B_{R}
\end{aligned}\right.
$$

where $f \in L^{q}\left(B_{R}\right)$ for some $q>1$.

Definition 3.2. We say that $u \in H_{0}^{1}\left(B_{R}\right), u \geq 0$ is a weak subsolution to problem (3.3) if

$$
\int_{B_{R}}\langle\nabla u, \nabla \varphi\rangle d \mu \leq \int_{B_{R}} f \varphi d \mu
$$

for any $\varphi \in H_{0}^{1}\left(B_{R}\right), \varphi \geq 0$.

In the next lemma, we recall [14, Lemma 3.6], which will be used later.

Lemma 3.3. Let $v \in L^{1}\left(B_{R}\right)$. Let $\bar{k}>0$. Suppose that there exist $C>0$ and $s>1$ such that

$$
g(k) \leq C \mu\left(A_{k}\right)^{s} \quad \text { for any } k \geq \bar{k}
$$

Then, $v \in L^{\infty}\left(B_{R}\right)$ and

$$
\|v\|_{L^{\infty}\left(B_{R}\right)} \leq \frac{s}{s-1} C^{\frac{1}{s}}\|v\|_{L^{1}\left(B_{R}\right)}^{1-\frac{1}{s}}+\bar{k}
$$

The following proposition contains an estimate in the spirit of the $L^{\infty}$ one of Stampacchia (see, e.g. $[4,24]$ and references therein) in the ball $B_{R}$; however, some differences are in order. In fact, we aim at obtaining an estimate independent of the radius $R$ (see Remark 3.5). Since the volume of $M$ is infinite, the classical estimate of Stampacchia cannot be directly applied.

Proposition 3.4. Let $f \in L^{m}\left(B_{R}\right)$ where $m>\frac{N}{2}$. Assume that $v \in H_{0}^{1}\left(B_{R}\right), v \geq 0$ is a subsolution to problem

$$
\left\{\begin{aligned}
-\Delta v=f & \text { in } B_{R}, \\
v=0 & \text { on } \partial B_{R},
\end{aligned}\right.
$$

in the sense of Definition 3.2. Then,

$$
\|v\|_{L^{\infty}\left(B_{R}\right)} \leq \frac{s}{s-1}\left(\frac{1}{C_{S}}\right)^{\frac{2}{s}}\|f\|_{L^{m}\left(B_{R}\right)}^{\frac{1}{s}}\|v\|_{L^{1}\left(B_{R}\right)}^{\frac{s-1}{s}},
$$

where

$$
s=1+\frac{2}{N}-\frac{1}{m}
$$


Remark 3.5. If in Proposition 3.4 we further assume that there exists a constant $k_{0}>0$ such that

$$
\max \left\{\|v\|_{L^{1}\left(B_{R}\right)},\|f\|_{L^{m}\left(B_{R}\right)}\right\} \leq k_{0} \quad \text { for all } R>0
$$

then from (3.5), we infer that the bound from above on $\|v\|_{L^{\infty}\left(B_{R}\right)}$ is independent of $R$. This fact will have a key role in the proof of global existence for problem (1.1).

Proof of Proposition 3.4. We define

$$
G_{k}(v):=v-T_{k}(v)
$$

where $T_{k}(v)$ has been defined in (3.2) and

$$
A_{k}:=\left\{x \in B_{R}:|v(x)|>k\right\} .
$$

Since $G_{k}(v) \in H_{0}^{1}\left(B_{R}\right)$ and $G_{k}(v) \geq 0$, we can take $G_{k}(v)$ as test function in problem (3.4). Arguing as in the proof of [14, Proposition 3.3], we obtain

$$
\int_{B_{R}}\left|G_{k}(v)\right| d \mu \leq \frac{1}{C_{S}^{2}}\|f\|_{L^{m}\left(B_{R}\right)} \mu\left(A_{k}\right)^{\frac{N+2}{N}-\frac{1}{m}} .
$$

By (3.6), setting

$$
C=\frac{1}{C_{S}^{2}}\|f\|_{L^{m}\left(B_{R}\right)}
$$

we rewrite 3.7 as

$$
\int_{B_{R}}\left|G_{k}(v)\right| d \mu \leq C \mu\left(A_{k}\right)^{s} .
$$

Hence, we can apply Lemma 3.3 to $v$ and we obtain

$$
\|v\|_{L^{\infty}\left(B_{R}\right)} \leq C^{\frac{1}{s}} \frac{s}{s-1}\|v\|_{L^{1}\left(B_{R}\right)}^{\frac{s-1}{s}}+\bar{k}
$$

Taking the limit as $\bar{k} \longrightarrow 0$ and we get the thesis.

We shall use the following Aronson-Benilan-type estimate (see [2]; see also [37, Proposition 2.3]).

Proposition 3.6. Let $m>1, p>m, u_{0} \in H_{0}^{1}\left(B_{R}\right) \cap L^{\infty}\left(B_{R}\right), u_{0} \geq 0$. Let $u$ be the solution to problem (3.2). Then, for a.e. $t \in(0, T)$,

$$
-\Delta u^{m}(\cdot, t) \leq u^{p}(\cdot, t)+\frac{1}{(m-1) t} u(\cdot, t) \text { in } \mathfrak{D}^{\prime}\left(B_{R}\right) .
$$


Proof. The conclusion follows by minor modifications of the proof of [37, Proposition 2.3] (where $p<m$ ), due to the fact that we have $p>m$. We define

$$
z=u_{t}+\frac{u}{m-1}
$$

and the operator

$$
L z=\Delta\left(m u^{m-1} z\right)+m u^{p-1} z
$$

where $u$ is the solution to problem (3.2). Observe that

$$
\begin{aligned}
& z(x, 0) \geq 0 \text { for } x \in B_{R}, \\
& z(x, t) \geq 0 \text { for } x \in \partial B_{R} \text { and } t \in(0, T) .
\end{aligned}
$$

Moreover, by direct computation, we get

$$
z_{t}-L z \geq 0 \text { in } B_{R} \times(0, T)
$$

Thus, arguing as in [37, Proposition 2.3], thanks to the comparison principle, we get, for a.e. $t \in(0, T)$,

$$
\begin{aligned}
& -\Delta u^{m}(\cdot, t) \leq T_{k}\left[u^{p}(\cdot, t)\right]+\frac{1}{(m-1) t} u(\cdot, t) \leq u^{p}(\cdot, t)+\frac{1}{(m-1) t} u(\cdot, t) \\
& \quad \text { in } \mathfrak{D}^{\prime}\left(B_{R}\right),
\end{aligned}
$$

where we have used that $T_{k}\left(u^{p}\right) \leq u^{p}$.

\section{4. $L^{q}$ and smoothing estimates for $p>m+\frac{2}{N}$}

Lemma 4.1. Let $m>1, p>m+\frac{2}{N}$. Assume that inequality (1.2) holds. Suppose that $u_{0} \in L^{\infty}\left(B_{R}\right), u_{0} \geq 0$. Let $1<q<\infty$, $p_{0}$ as in (2.1) and assume that

$$
\left\|u_{0}\right\|_{L^{p_{0}\left(B_{R}\right)}}<\bar{\varepsilon}
$$

with $\bar{\varepsilon}=\bar{\varepsilon}\left(p, m, q, C_{s}\right)$ sufficiently small. Let $u$ be the solution of problem (3.2) in the sense of Definition 3.1, such that in addition $u \in C\left([0, T), L^{q}\left(B_{R}\right)\right)$ for any $q \in$ $(1,+\infty)$, for any $T>0$. Then,

$$
\|u(t)\|_{L^{q}\left(B_{R}\right)} \leq\left\|u_{0}\right\|_{L^{q}\left(B_{R}\right)} \text { for all } t>0 .
$$

Note that the request $u \in C\left([0, T), L^{q}\left(B_{R}\right)\right)$ for any $q \in(1,+\infty)$, for any $T>0$ is not restrictive, since we will construct solutions belonging to that class (see the proof of Theorem 2.2 below). This remark also applies to several other intermediate results below. 
Proof. Since $u_{0}$ is bounded and $T_{k}$ is a bounded and Lipschitz function, by standard results, there exists a unique solution of problem (3.2) in the sense of Definition 3.1. We now multiply both sides of the differential equation in problem (3.2) by $u^{q-1}$,

$$
\int_{B_{R}} u_{t} u^{q-1} d \mu=\int_{B_{R}} \Delta\left(u^{m}\right) u^{q-1} d \mu+\int_{B_{R}} T_{k}\left(u^{p}\right) u^{q-1} d \mu .
$$

Now, formally integrating by parts in $B_{R}$. This can be justified by standard tools, by an approximation procedure. We get

$$
\begin{aligned}
& \frac{1}{q} \frac{d}{d t} \int_{B_{R}} u^{q} d \mu=-m(q-1) \int_{B_{R}} u^{m+q-3}|\nabla u|^{2} d \mu \\
& \quad+\int_{B_{R}} T_{k}\left(u^{p}\right) u^{q-1} d \mu
\end{aligned}
$$

Observe that, thanks to Sobolev inequality (1.2), we have

$$
\begin{aligned}
\int_{B_{R}} u^{m+q-3}|\nabla u|^{2} d \mu & =\frac{4}{(m+q-1)^{2}} \int_{B_{R}}\left|\nabla\left(u^{\frac{m+q-1}{2}}\right)\right|^{2} d \mu \\
& \geq \frac{4}{(m+q-1)^{2}} C_{s}^{2}\left(\int_{B_{R}} u^{\frac{m+q-1}{2} \frac{2 N}{N-2}} d \mu\right)^{\frac{N-2}{N}} .
\end{aligned}
$$

Moreover, the last term in the right-hand side of (4.3), thanks to Hölder inequality with exponents $\frac{N}{N-2}$ and $\frac{N}{2}$, becomes

$$
\begin{aligned}
\int_{B_{R}} T_{k}\left(u^{p}\right) u^{q-1} d \mu & \leq \int_{B_{R}} u^{p} u^{q-1} d \mu=\int_{B_{R}} u^{p-m} u^{m+q-1} d \mu \\
& \leq\|u(t)\|_{L^{(p-m) \frac{N}{2}\left(B_{R}\right)}}^{\|u(t)\|_{L^{(m+q-1)} \frac{N}{N-2}\left(B_{R}\right)}^{m+q-1}} .
\end{aligned}
$$

Combining (4.4) and (4.5), we get

$\frac{1}{q} \frac{d}{d t}\|u(t)\|_{L^{q}\left(B_{R}\right)}^{q} \leq-\left[\frac{4 m(q-1)}{(m+q-1)^{2}} C_{s}^{2}-\|u(t)\|_{L^{p_{0}\left(B_{R}\right)}}^{p-m}\right]\|u(t)\|_{L^{(m+q-1)} \frac{N}{N-2}\left(B_{R}\right)}^{m+q-1}$.

Take any $T>0$. Observe that, thanks to hypothesis (4.1) and the known continuity of the map $t \mapsto u(t)$ in $[0, T]$, there exists $t_{0}>0$ such that

$$
\|u(t)\|_{L^{p_{0}\left(B_{R}\right)}} \leq 2 \bar{\varepsilon} \text { for any } t \in\left[0, t_{0}\right] .
$$

Hence, (4.6) becomes, for any $t \in\left(0, t_{0}\right]$,

$$
\frac{1}{q} \frac{d}{d t}\|u(t)\|_{L^{q}\left(B_{R}\right)}^{q} \leq-\left[\frac{4 m(q-1)}{(m+q-1)^{2}} C_{s}^{2}-2 \bar{\varepsilon}^{p-m}\right]\|u(t)\|_{L^{(m+q-1)} \frac{N}{N-2}\left(B_{R}\right)}^{m+q-1} \leq 0,
$$


where the last inequality is obtained thanks to (4.1). We have proved that $t \mapsto$ $\|u(t)\|_{L^{q}\left(B_{R}\right)}$ is decreasing in time for any $t \in\left(0, t_{0}\right]$, i.e.

$$
\|u(t)\|_{L^{q}\left(B_{R}\right)} \leq\left\|u_{0}\right\|_{L^{q}\left(B_{R}\right)} \text { for any } t \in\left(0, t_{0}\right]
$$

In particular, inequality (4.7) follows for the choice $q=p_{0}$, in view of hypothesis (4.1). Hence, we have

$$
\|u(t)\|_{L^{p_{0}\left(B_{R}\right)}} \leq\left\|u_{0}\right\|_{L^{p_{0}\left(B_{R}\right)}}<\bar{\varepsilon} \text { for any } t \in\left(0, t_{0}\right] .
$$

Now, we can repeat the same argument in the time interval $\left(t_{0}, t_{1}\right]$, where $t_{1}$ is chosen, due to the continuity of $u$, in such a way that

$$
\|u(t)\|_{L^{p_{0}\left(B_{R}\right)}} \leq 2 \bar{\varepsilon} \quad \text { for any } t \in\left(t_{0}, t_{1}\right] .
$$

Thus, we get

$$
\|u(t)\|_{L^{q}\left(B_{R}\right)} \leq\left\|u_{0}\right\|_{L^{q}\left(B_{R}\right)} \text { for any } t \in\left(0, t_{1}\right]
$$

Iterating this procedure, we obtain that $t \mapsto\|u(t)\|_{L^{q}\left(B_{R}\right)}$ is decreasing in $[0, T]$. Since $T>0$ was arbitrary, the thesis follows.

Using a Moser-type iteration procedure, we prove the following result:

Proposition 4.2. Let $m>1, p>m+\frac{2}{N}$. Assume that inequality (1.2) holds. Suppose that $u_{0} \in L^{\infty}\left(B_{R}\right), u_{0} \geq 0$. Let $u$ be the solution of problem (3.2) in the sense of Definition 3.1, such that in addition $u \in C\left([0, T), L^{q}\left(B_{R}\right)\right)$ for any $q \in$ $(1,+\infty)$, for any $T>0$. Let $1<q_{0} \leq q<+\infty$ and assume that

$$
\left\|u_{0}\right\|_{L^{p_{0}\left(B_{R}\right)}}<\tilde{\varepsilon}_{0}
$$

for $\tilde{\varepsilon}_{0}=\tilde{\varepsilon}_{0}\left(p, m, N, C_{s}, q, q_{0}\right)$ sufficiently small. Then, there exists $C\left(m, q_{0}, C_{s}, \tilde{\varepsilon}_{0}\right.$, $N, q)>0$ such that

$$
\|u(t)\|_{L^{q}\left(B_{R}\right)} \leq C t^{-\gamma_{q}}\left\|u_{0}\right\|_{L^{q_{0}\left(B_{R}\right)}}^{\delta_{q}} \text { for all } t>0
$$

where

$$
\gamma_{q}=\left(\frac{1}{q_{0}}-\frac{1}{q}\right) \frac{N q_{0}}{2 q_{0}+N(m-1)}, \quad \delta_{q}=\frac{q_{0}}{q}\left(\frac{q+\frac{N}{2}(m-1)}{q_{0}+\frac{N}{2}(m-1)}\right) .
$$

Proof. Let $\left\{q_{n}\right\}$ be the sequence defined in (2.7). We start by proving a smoothing estimate from $q_{0}$ to $q_{\bar{n}}$ using a Moser iteration technique (see also [1]).

Let $t>0$, we define

$$
s=\frac{t}{2^{\bar{n}}-1}, \quad t_{n}=\left(2^{n}-1\right) s .
$$


Observe that $t_{0}=0, \quad t_{\bar{n}}=t, \quad\left\{t_{n}\right\}$ is an increasing sequence w.r.t. $n$. Now, for any $1 \leq n \leq \bar{n}$, we multiply equation (3.2) by $u^{q_{n-1}-1}$ and integrate in $B_{R} \times\left[t_{n-1}, t_{n}\right]$. Thus, we get

$$
\begin{aligned}
& \int_{t_{n-1}}^{t_{n}} \int_{B_{R}} u_{t} u^{q_{n-1}-1} d \mu d t=\int_{t_{n-1}}^{t_{n}} \int_{B_{R}} \Delta\left(u^{m}\right) u^{q_{n-1}-1} d \mu d t \\
& \quad+\int_{t_{n-1}}^{t_{n}} \int_{B_{R}} T_{k}\left(u^{p}\right) u^{q_{n-1}-1} d \mu d t .
\end{aligned}
$$

Then, we integrate by parts in $B_{R} \times\left[t_{n-1}, t_{n}\right]$. Thanks to Sobolev inequality and hypothesis (4.8), we get

$$
\begin{aligned}
& \frac{1}{q_{n-1}}\left[\left\|u\left(\cdot, t_{n}\right)\right\|_{L^{q_{n-1}\left(B_{R}\right)}}^{q_{n-1}}-\left\|u\left(\cdot, t_{n-1}\right)\right\|_{L^{q_{n-1}\left(B_{R}\right)}}^{q_{n-1}}\right] \\
& \leq-\left[\frac{4 m\left(q_{n-1}-1\right)}{\left(m+q_{n-1}-1\right)^{2}} C_{s}^{2}-2 \tilde{\varepsilon}_{0}^{\frac{1}{p-m}}\right] \int_{t_{n-1}}^{t_{n}}\|u(\tau)\|_{L^{\left(m+q_{n-1}-1\right)} \frac{N}{N-2}\left(B_{R}\right)}^{m+q_{n-1}-1} d \tau,
\end{aligned}
$$

where we have used the fact that $T_{k}\left(u^{p}\right) \leq u^{p}$. We define $q_{n}$ as in (2.7), so that $(m+$ $\left.q_{n-1}-1\right) \frac{N}{N-2}=q_{n}$. Hence, in view of hypothesis (4.8) we can apply Lemma 4.1 to the integral on the right-hand side of (4.11), hence we get

$$
\begin{aligned}
& \frac{1}{q_{n-1}}\left[\left\|u\left(\cdot, t_{n}\right)\right\|_{L^{q_{n-1}\left(B_{R}\right)}}^{q_{n-1}}-\left\|u\left(\cdot, t_{n-1}\right)\right\|_{L^{q_{n-1}\left(B_{R}\right)}}^{q_{n-1}}\right] \\
& \leq-\left[\frac{4 m\left(q_{n-1}-1\right)}{\left(m+q_{n-1}-1\right)^{2}} C_{s}^{2}-2 \tilde{\varepsilon}_{0}^{\frac{1}{p-m}}\right]\left\|u\left(\cdot, t_{n}\right)\right\|_{L^{q_{n}\left(B_{R}\right)}}^{m+q_{n-1}-1}\left|t_{n}-t_{n-1}\right| .
\end{aligned}
$$

Observe that

$$
\begin{gathered}
\left\|u\left(\cdot, t_{n}\right)\right\|_{L^{q_{n-1}\left(B_{R}\right)}}^{q_{n-1}} \geq 0, \\
\left|t_{n}-t_{n-1}\right|=\frac{2^{n-1} t}{2^{\bar{n}}-1} .
\end{gathered}
$$

We define

$$
d_{n-1}:=\left[\frac{4 m\left(q_{n-1}-1\right)}{\left(m+q_{n-1}-1\right)^{2}} C_{s}^{2}-2 \tilde{\varepsilon}_{0}^{\frac{1}{p-m}}\right]^{-1} \frac{1}{q_{n-1}} .
$$

By plugging (4.13) and (4.14) into (4.12), we get

$$
\left\|u\left(\cdot, t_{n}\right)\right\|_{L^{q_{n}\left(B_{R}\right)}}^{m+q_{n-1}-1} \leq \frac{\left(2^{\bar{n}}-1\right) d_{n}}{2^{n-1} t}\left\|u\left(\cdot, t_{n-1}\right)\right\|_{L^{q_{n-1}\left(B_{R}\right)}}^{q_{n-1}} .
$$

The latter formula can be rewritten as

$$
\left\|u\left(\cdot, t_{n}\right)\right\|_{L^{q_{n}}\left(B_{R}\right)} \leq\left(\frac{\left(2^{\bar{n}}-1\right) d_{n}}{2^{n-1}}\right)^{\frac{1}{m+q_{n-1}-1}} t^{-\frac{1}{m+q_{n-1}-1}}\left\|u\left(\cdot, t_{n-1}\right)\right\|_{L^{q_{n-1}\left(B_{R}\right)}}^{\frac{q_{n-1}}{m+q_{n-1}-1}} .
$$


Thanks to the definition of the sequence $\left\{q_{n}\right\}$ in (2.7), we write

$$
\left\|u\left(\cdot, t_{n}\right)\right\|_{L^{q_{n}}\left(B_{R}\right)} \leq\left(\frac{\left(2^{\bar{n}}-1\right) d_{n-1}}{2^{n-1}}\right)^{\frac{N}{(N-2)} \frac{1}{q_{n}}} t^{-\frac{N}{(N-2)} \frac{1}{q_{n}}}\left\|u\left(\cdot, t_{n-1}\right)\right\|_{L^{\frac{q_{n-1}}{q_{n}} \frac{N}{N-2}\left(B_{R}\right)}} .
$$

Define $\sigma:=\frac{N}{N-2}$. Observe that, for any $1 \leq n \leq \bar{n}$, we have

$$
\begin{aligned}
\left(\frac{\left(2^{\bar{n}}-1\right) d_{n-1}}{2^{n-1}}\right)^{\sigma} & =\left[\frac{2^{\bar{n}}-1}{2^{n-1}}\left(\frac{4 m\left(q_{n-1}-1\right)}{\left(m+q_{n-1}-1\right)^{2}} C_{s}^{2}-2 \varepsilon^{\frac{1}{p-m}}\right)^{-1} \frac{1}{q_{n-1}}\right]^{\sigma} \\
& =\left[\frac{2^{\bar{n}}-1}{2^{n-1}} \frac{1}{\frac{4 m q_{n-1}\left(q_{n-1}-1\right)}{\left(m+q_{n-1}-1\right)^{2}} C_{s}^{2}-2 \tilde{\varepsilon}_{0}^{\frac{1}{p-m}} q_{n-1}}\right]^{\sigma},
\end{aligned}
$$

where

$$
\frac{2^{\bar{n}}-1}{2^{n-1}} \leq 2^{\bar{n}+1} \quad \text { for all } 1 \leq n \leq \bar{n}
$$

Consider the function

$$
g(x):=\left[\frac{4 m(x-1)}{(m+x-1)^{2}} C_{s}^{2}-2 \tilde{\varepsilon}_{0}^{\frac{1}{p-m}}\right] x \quad \text { for } q_{0} \leq x \leq q_{\bar{n}}, \quad x \in \mathbb{R} .
$$

Observe that, thanks to the definition of $\sigma, g(x)>0$ for any $q_{0} \leq x \leq q_{\bar{n}}$. Moreover, $g$ has a minimum in the interval $q_{0} \leq x \leq q_{\bar{n}}$, call it $\tilde{x}$. Then, we have

$$
\frac{1}{g(x)} \leq \frac{1}{g(\tilde{x})} \quad \text { for any } \quad q_{0} \leq x \leq q_{\bar{n}}, x \in \mathbb{R} .
$$

Thanks to (4.16), (4.17) and (4.18), we can say that there exist a positive constant $C$, where $C=C\left(N, C_{s}, \varepsilon, \bar{n}, m, q_{0}\right)$, such that

$$
\left(\frac{\left(2^{\bar{n}}-1\right) d_{n-1}}{2^{n-1}}\right)^{\sigma} \leq C, \text { for all } 1 \leq n \leq \bar{n}
$$

By using (4.19) and (4.15), we get, for any $1 \leq n \leq \bar{n}$

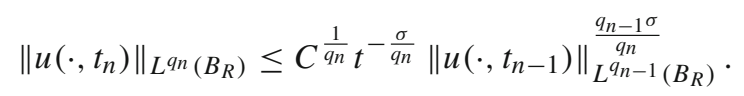

Let us set

$$
U_{n}:=\left\|u\left(\cdot, t_{n}\right)\right\|_{L^{q_{n}\left(B_{R}\right)}} .
$$


Then, (4.20) becomes

$$
\begin{aligned}
U_{n} & \leq C^{\frac{1}{q_{n}}} t^{-\frac{\sigma}{q_{n}}} U_{n-1}^{\frac{q_{n-1} \sigma}{q_{n}}} \\
& \leq C^{\frac{1}{q_{n}}} t^{-\frac{\sigma}{q_{n}}}\left[C^{\frac{\sigma}{q_{n}}} t^{-\frac{\sigma^{2}}{q_{n}}} U_{k-2}^{\sigma^{2} \frac{q_{n-2}}{q_{n}}}\right] \\
& \leq \cdots \\
& \leq C^{\frac{1}{q_{n}} \sum_{i=0}^{n-1} \sigma^{i}} t^{-\frac{\sigma}{q_{n}} \sum_{i=0}^{n-1} \sigma^{i}} U_{0}^{\sigma^{n} \frac{q_{0}}{q_{n}}} .
\end{aligned}
$$

We define

$$
\alpha_{n}:=\frac{1}{q_{n}} \sum_{i=0}^{n-1} \sigma^{i}, \quad \beta_{n}:=\frac{\sigma}{q_{n}} \sum_{i=0}^{n-1} \sigma^{i}=\sigma \alpha_{n}, \quad \delta_{n}:=\sigma^{n} \frac{q_{0}}{q_{n}} .
$$

By substituting $n$ with $\bar{n}$ into (4.21), we get

$$
\alpha_{\bar{n}}:=\frac{N-2}{2} \frac{A}{q_{\bar{n}}}, \quad \beta_{\bar{n}}:=\frac{N}{2} \frac{A}{q_{\bar{n}}}, \quad \delta_{\bar{n}}:=(A+1) \frac{q_{0}}{q_{\bar{n}}},
$$

where $A:=\left(\frac{N}{N-2}\right)^{\bar{n}}-1$. Hence, in view of (4.10) and (4.22), (4.20) with $n=\bar{n}$ yields

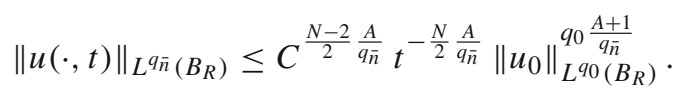

We have proved a smoothing estimate from $q_{0}$ to $q_{\bar{n}}$. Observe that if $q_{\bar{n}}=q$ then the thesis is proved. Now suppose that $q>q_{\bar{n}}$. Observe that $q_{0} \leq q<q_{\bar{n}}$ and define

$$
B:=N(m-1) A+2 q_{0}(A+1)
$$

From (4.23) and Lemma 4.1, we get, by interpolation,

$$
\begin{aligned}
\|u(\cdot, t)\|_{L^{q}\left(B_{R}\right)} & \leq\|u(\cdot, t)\|_{L^{q_{0}\left(B_{R}\right)}}^{\theta}\|u(\cdot, t)\|_{L^{q_{\bar{n}}\left(B_{R}\right)}}^{1-\theta} \\
& \leq\left\|u_{0}(\cdot)\right\|_{L^{q_{0}\left(B_{R}\right)}}^{\theta} C t^{-\frac{N A}{B}(1-\theta)}\left\|u_{0}\right\|_{L^{q_{0}\left(B_{R}\right)}}^{2 q_{0} \frac{A+1}{B}(1-\theta)} \\
& =C t^{-\frac{N A}{B}(1-\theta)}\left\|u_{0}\right\|_{L^{q_{0}}\left(B_{R}\right)}^{2 q_{0} \frac{A+1}{B}(1-\theta)+\theta}
\end{aligned}
$$

where

$$
\theta=\frac{q_{0}}{q}\left(\frac{q_{\bar{n}}-q}{q_{\bar{n}}-q_{0}}\right) .
$$

Combining (4.24), (4.9) and (4.25), we get the claim, noticing that $q$ was arbitrary in $\left[q_{0}, \infty\right)$. 
Remark 4.3. One cannot let $q \rightarrow+\infty$ in the above bound. In fact, one can show that $\varepsilon \longrightarrow 0$ as $q \rightarrow \infty$. So in such limit the hypothesis on the norm of the initial datum (2.2) is satisfied only when $u_{0} \equiv 0$.

Proposition 4.4. Let $m>1, p>m+\frac{2}{N}, R>0, p_{0}$ be as in (2.1), $u_{0} \in L^{\infty}\left(B_{R}\right)$, $u_{0} \geq 0$. Let

$$
r>\max \left\{p_{0}, \frac{N}{2}\right\}, \quad s=1+\frac{2}{N}-\frac{1}{r} .
$$

Suppose that (2.2) holds for $\varepsilon_{0}=\varepsilon_{0}\left(p, m, N, C_{s}, r\right)$ sufficiently small. Let $u$ be the solution to problem (3.2), such that in addition $u \in C\left([0, T), L^{q}\left(B_{R}\right)\right)$ for any $q \in$ $(1,+\infty)$, for any $T>0$. Let $M$ be such that inequality (1.2) holds. Then, there exists $\Gamma=\Gamma(p, m, N, r)>0$ such that, for all $t>0$,

$$
\|u(t)\|_{L^{\infty}\left(B_{R}\right)} \leq \Gamma t^{-\frac{\gamma}{m s}}\left\{\left\|u_{0}\right\|_{L^{p_{0}\left(B_{R}\right)}}^{\delta_{1}}+\frac{1}{m-1}\left\|u_{0}\right\|_{L^{p_{0}\left(B_{R}\right)}}^{\delta_{2}}\right\}^{\frac{1}{m s}}\left\|u_{0}\right\|_{L^{m}\left(B_{R}\right)}^{\frac{s-1}{s}},
$$

where

$$
\begin{aligned}
& \gamma=\frac{p}{p-1}\left[1-\frac{N(p-m)}{2 p r}\right], \delta_{1}=p \frac{p-m}{m-1}\left[1+\frac{N(m-1)}{2 p r}\right], \\
& \delta_{2}=\frac{p-m}{m-1}\left[1+\frac{N(m-1)}{2 r}\right] .
\end{aligned}
$$

Remark 4.5. If in Proposition 4.4, in addition, we assume that for some $k_{0}>0$

$$
\max \left\{\left\|u_{0}\right\|_{L^{m}\left(B_{R}\right)} ;\left\|u_{0}\right\|_{L^{p_{0}\left(B_{R}\right)}}\right\} \leq k_{0} \text { for every } R>0,
$$

then the bound from above for $\|u(t)\|_{L^{\infty}\left(B_{R}\right)}$ in (4.27) is independent of $R$.

Proof of Proposition 4.4. Let us set $w=u(\cdot, t)$. Observe that $w^{m} \in H_{0}^{1}\left(B_{R}\right)$ and $w \geq 0$. Due to Proposition 3.6, we know that

$$
-\Delta\left(w^{m}\right) \leq\left[w^{p}+\frac{w}{(m-1) t}\right] .
$$

Observe that, since $u_{0} \in L^{\infty}\left(B_{R}\right)$ also $w \in L^{\infty}\left(B_{R}\right)$. Due to (4.26), we can apply Proposition 3.4. So, we have that

$$
\begin{aligned}
\|w\|_{L^{\infty}\left(B_{R}\right)}^{m} & \leq \frac{s}{s-1}\left(\frac{1}{C_{s}}\right)^{\frac{2}{s}}\left\|w^{p}+\frac{w}{(m-1) t}\right\|_{L^{r}\left(B_{R}\right)}^{\frac{1}{s}}\left\|w^{m}\right\|_{L^{1}\left(B_{R}\right)}^{\frac{s-1}{s}} \\
& \leq \frac{s}{s-1}\left(\frac{1}{C_{s}}\right)^{\frac{2}{s}}\left\{\left\|w^{p}\right\|_{L^{r}\left(B_{R}\right)}+\frac{1}{(m-1) t}\|w\|_{L^{r}\left(B_{R}\right)}\right\}^{\frac{1}{s}}\|w\|_{L^{m}\left(B_{R}\right)}^{m \frac{s-1}{s}}
\end{aligned}
$$


where $s$ has been defined in (3.6). Thanks to (2.2), with an appropriate choice of $\varepsilon_{0}$, and (4.26) we can apply Proposition 4.2 with

$$
q=p r, \quad q_{0}=p_{0}, \quad \gamma_{p r}=\frac{1}{p-1}\left[1-\frac{N(p-m)}{2 p r}\right]
$$

and $\delta_{p r}=\delta_{1} / p, \delta_{1}$ defined in (4.28). Hence, we obtain

$$
\left\|w^{p}\right\|_{L^{r}\left(B_{R}\right)}=\|w\|_{L^{p r}\left(B_{R}\right)}^{p} \leq\left[C t^{-\gamma_{p r}}\left\|u_{0}\right\|_{L^{p_{0}\left(B_{R}\right)}}^{\delta_{1} / p}\right]^{p}
$$

where $C>0$ is defined in Proposition 4.2. Similarly, by (2.2), with an appropriate choice of $\varepsilon_{0}$, and (4.26), we can apply Proposition 4.2 with

$$
q=r, \quad q_{0}=p_{0}, \quad \gamma_{r}=\frac{1}{p-1}\left[1-\frac{N(p-m)}{2 r}\right]
$$

and $\delta_{r}=\delta_{2}$ as defined in (4.28). Hence, we obtain

$$
\|w\|_{L^{r}\left(B_{R}\right)} \leq C t^{-\gamma_{r}}\left\|u_{0}\right\|_{L^{p_{0}\left(B_{R}\right)}}^{\delta_{2}},
$$

where $C>0$ is defined in Proposition 4.2. Plugging (4.30) and (4.31) into (4.29), we obtain

$$
\begin{aligned}
& \|w\|_{L^{\infty}\left(B_{R}\right)}^{m} \leq \frac{s}{s-1}\left(\frac{1}{C_{s}}\right)^{\frac{2}{s}}\left\{\left\|w^{p}\right\|_{L^{r}\left(B_{R}\right)}+\frac{1}{(m-1) t}\|w\|_{L^{r}\left(B_{R}\right)}\right\}^{\frac{1}{s}}\|w\|_{L^{m}\left(B_{R}\right)}^{m \frac{s-1}{s}} \\
& \leq \frac{s}{s-1}\left(\frac{1}{C_{s}}\right)^{\frac{2}{s}}\left\{C^{p} t^{-p \gamma_{p r}}\left\|u_{0}\right\|_{L^{p_{0}\left(B_{R}\right)}}^{\delta_{1}}+\frac{1}{(m-1) t} C t^{-\gamma_{r}}\left\|u_{0}\right\|_{L^{p_{0}\left(B_{R}\right)}}^{\delta_{2}}\right\}^{\frac{1}{s}}\|w\|_{L^{m}\left(B_{R}\right)}^{m \frac{s-1}{s}} .
\end{aligned}
$$

Observe that $-p \gamma_{p r}=-\gamma_{r}-1=\gamma$, where $\gamma$ has been defined in (4.28). Hence, we obtain

$$
\begin{aligned}
& \|w\|_{L^{\infty}\left(B_{R}\right)}^{m} \leq \frac{s}{s-1}\left(\frac{1}{C_{s}}\right)^{\frac{2}{s}} t^{-\frac{\gamma}{s}} \\
& \left\{C^{p}\left\|u_{0}\right\|_{L^{p_{0}\left(B_{R}\right)}}^{\delta_{1}}+\frac{1}{m-1} C\left\|u_{0}\right\|_{L^{p_{0}\left(B_{R}\right)}}^{\delta_{2}}\right\}^{\frac{1}{s}}\|w\|_{L^{m}\left(B_{R}\right)}^{m} .
\end{aligned}
$$

Moreover, since $u_{0} \in L^{\infty}\left(B_{R}\right)$, we can apply Lemma 4.1 to $w$ with $q=m$. Thus, from (4.2) with $q=m$ we get

$$
\begin{aligned}
& \|w\|_{L^{\infty}\left(B_{R}\right)}^{m} \leq \frac{s}{s-1}\left(\frac{1}{C_{s}}\right)^{\frac{2}{s}} t^{-\frac{\gamma}{s}} \\
& \left\{C^{p}\left\|u_{0}\right\|_{L^{p_{0}\left(B_{R}\right)}}^{\delta_{1}}+\frac{1}{m-1} C\left\|u_{0}\right\|_{L^{p_{0}\left(B_{R}\right)}}^{\delta_{2}}\right\}^{\frac{1}{s}}\left\|u_{0}\right\|_{L^{m}\left(B_{R}\right)}^{m \frac{s-1}{s}}
\end{aligned}
$$

Finally, define

$$
\Gamma:=\left[\frac{s}{s-1}\left(\frac{1}{C_{s}}\right)^{\frac{2}{s}} \max \left\{C^{\frac{p}{s}} ; C^{\frac{1}{s}}\right\}\right]^{\frac{1}{m}} .
$$


Hence, we obtain

$$
\|w\|_{L^{\infty}\left(B_{R}\right)} \leq \Gamma t^{-\frac{\gamma}{m s}}\left\{\left\|u_{0}\right\|_{L^{p_{0}\left(B_{R}\right)}}^{\delta_{1}}+\frac{1}{m-1}\left\|u_{0}\right\|_{L^{p_{0}\left(B_{R}\right)}}^{\delta_{2}}\right\}^{\frac{1}{m s}}\left\|u_{0}\right\|_{L^{m}\left(B_{R}\right)}^{\frac{s-1}{s}}
$$

\section{Proof of Theorem 2.2}

Proof of Theorem 2.2. Let $\left\{u_{0, h}\right\}_{h \geq 0}$ be a sequence of functions such that

(a) $u_{0, h} \in L^{\infty}(M) \cap C_{c}^{\infty}(M)$ for all $h \geq 0$,

(b) $u_{0, h} \geq 0$ for all $h \geq 0$,

(c) $u_{0, h_{1}} \leq u_{0, h_{2}}$ for any $h_{1}<h_{2}$,

(d) $u_{0, h} \longrightarrow u_{0}$ in $L^{m}(M) \cap L^{p_{0}}(M)$ as $h \rightarrow+\infty$,

where $p_{0}$ has been defined in (2.1). Observe that, due to assumptions $(c)$ and $(d), u_{0, h}$ satisfies (2.2). For any $R>0, k>0, h>0$, consider the problem

$$
\begin{cases}u_{t}=\Delta u^{m}+T_{k}\left(u^{p}\right) & \text { in } B_{R} \times(0,+\infty) \\ u=0 & \text { in } \partial B_{R} \times(0, \infty) \\ u=u_{0, h} & \text { in } B_{R} \times\{0\}\end{cases}
$$

From standard results, it follows that problem (5.1) has a solution $u_{h, k}^{R}$ in the sense of Definition 3.1; moreover, $u_{h, k}^{R} \in C\left([0, T] ; L^{q}\left(B_{R}\right)\right)$ for any $q>1$. Hence, by Lemma 4.1, in Proposition 4.2 and in Proposition 4.4, we have for any $t \in(0,+\infty)$,

$$
\begin{aligned}
& \left\|u_{h, k}^{R}(t)\right\|_{L^{m}\left(B_{R}\right)} \leq\left\|u_{0, h}\right\|_{L^{m}\left(B_{R}\right)} \\
& \left\|u_{h, k}^{R}(t)\right\|_{L^{p}\left(B_{R}\right)} \leq C t^{-\gamma_{p}}\left\|u_{0, h}\right\|_{L^{p_{0}\left(B_{R}\right)}}^{\delta_{p}}
\end{aligned}
$$

where

$$
\begin{gathered}
\gamma_{p}=\frac{1}{p-1}\left[1-\frac{N(p-m)}{2 p}\right], \quad \delta_{p}=\frac{p-m}{p-1}\left[1+\frac{N(m-1)}{2 p}\right], \\
\left\|u_{h, k}^{R}\right\|_{L^{\infty}\left(B_{R}\right)} \leq \Gamma t^{-\frac{\gamma}{m s}}\left\{\left\|u_{0, h}\right\|_{L^{p_{0}\left(B_{R}\right)}}^{\delta_{1}}+\frac{1}{m-1}\left\|u_{0, h}\right\|_{L^{p_{0}\left(B_{R}\right)}}^{\delta_{2}}\right\}^{\frac{1}{m s}}\left\|u_{0, h}\right\|_{L^{m}\left(B_{R}\right)}^{\frac{s-1}{s}},
\end{gathered}
$$


with $s$ as in (4.26) and $\gamma, \delta_{1}, \delta_{2}$ as in (4.28). In addition, for any $\tau \in(0, T), \zeta \in$ $C_{c}^{1}((\tau, T)), \zeta \geq 0, \max _{[\tau, T]} \zeta^{\prime}>0$,

$$
\begin{aligned}
& \int_{\tau}^{T} \zeta(t)\left[\left(\left(u_{h, k}^{R}\right)^{\frac{m+1}{2}}\right)_{t}\right]^{2} d \mu d t \leq \max _{[\tau, T]} \zeta^{\prime} \bar{C} \int_{B_{R}}\left(u_{h, k}^{R}\right)^{m+1}(x, \tau) d \mu \\
& \quad+\bar{C} \max _{[\tau, T]} \zeta \int_{B_{R}} F\left(u_{h, k}^{R}(x, T)\right) d \mu \\
& \quad \leq \max _{[\tau, T]} \zeta^{\prime}(t) \bar{C}\left\|u_{h, k}^{R}(\tau)\right\|_{L^{\infty}\left(B_{R}\right)}\left\|u_{h, k}^{R}(\tau)\right\|_{L^{m}\left(B_{R}\right)}^{m} \\
& \quad+\frac{\bar{C}}{m+p}\left\|u_{h, k}^{R}(T)\right\|_{L^{\infty}\left(B_{R}\right)}^{p}\left\|u_{h, k}^{R}(T)\right\|_{L^{m}\left(B_{R}\right)}^{m}
\end{aligned}
$$

where

$$
F(u)=\int_{0}^{u} s^{m-1+p} d s,
$$

and $\bar{C}>0$ is a constant only depending on $m$. Inequality (5.5) is formally obtained by multiplying the differential inequality in problem (3.2) by $\zeta(t)\left[\left(u^{m}\right)_{t}\right]$, and integrating by parts; indeed, a standard approximation procedure is needed (see [17, Lemma 3.3] and [3, Theorem 13]).

Moreover, as a consequence of Definition 3.1, for any $\varphi \in C_{c}^{\infty}\left(B_{R} \times[0, T]\right)$ such that $\varphi(x, T)=0$ for any $x \in B_{R}, u_{h, k}^{R}$ satisfies

$$
\begin{aligned}
-\int_{0}^{T} \int_{B_{R}} u_{h, k}^{R} \varphi_{t} d \mu d t= & \int_{0}^{T} \int_{B_{R}}\left(u_{h, k}^{R}\right)^{m} \Delta \varphi d \mu d t+\int_{0}^{T} \int_{B_{R}} T_{k}\left[\left(u_{h, k}^{R}\right)^{p}\right] \varphi d \mu d t \\
& +\int_{B_{R}} u_{0, h}(x) \varphi(x, 0) d \mu
\end{aligned}
$$

where all the integrals are finite. Now, observe that, for any $h>0$ and $R>0$ the sequence of solutions $\left\{u_{h, k}^{R}\right\}_{k \geq 0}$ is monotone increasing in $k$ hence it has a pointwise limit for $k \rightarrow \infty$. Let $u_{h}^{R}$ be such limit so that we have

$$
u_{h, k}^{R} \longrightarrow u_{h}^{R} \text { as } k \rightarrow \infty \text { pointwise. }
$$

In view of (5.2), (5.3) and (5.4), the right-hand side of (5.5) is independent of $k$. So, $\left(u_{h}^{R}\right)^{\frac{m+1}{2}} \in H^{1}\left((\tau, T) ; L^{2}\left(B_{R}\right)\right)$. Therefore, $\left(u_{h}^{R}\right)^{\frac{m+1}{2}} \in C\left([\tau, T] ; L^{2}\left(B_{R}\right)\right)$. We can now pass to the limit as $k \rightarrow+\infty$ in inequalities (5.2), (5.3) and (5.4) arguing as follows. From inequality (5.2) and (5.3), thanks to the Fatou's Lemma, one has for all $t>0$

$$
\begin{aligned}
\left\|u_{h}^{R}(t)\right\|_{L^{m}\left(B_{R}\right)} \leq & \left\|u_{0, h}\right\|_{L^{m}\left(B_{R}\right)} . \\
& \left\|u_{h}^{R}(t)\right\|_{L^{p}\left(B_{R}\right)} \leq C t^{-\gamma_{p}}\left\|u_{0, h}\right\|_{L^{p_{0}\left(B_{R}\right)}}^{\delta_{p}} ;
\end{aligned}
$$


On the other hand, from (5.4), since $u_{h, k}^{R} \longrightarrow u_{h}^{R}$ as $k \rightarrow \infty$ pointwise and the right-hand side of (5.4) is independent of $k$, one has for all $t>0$

$$
\left\|u_{h}^{R}\right\|_{L^{\infty}\left(B_{R}\right)} \leq \Gamma t^{-\frac{\gamma}{m s}}\left\{\left\|u_{0, h}\right\|_{L^{p_{0}\left(B_{R}\right)}}^{\delta_{1}}+\frac{1}{m-1}\left\|u_{0, h}\right\|_{L^{p_{0}\left(B_{R}\right)}}^{\delta_{2}}\right\}^{\frac{1}{m s}}\left\|u_{0, h}\right\|_{L^{m}\left(B_{R}\right), 9}^{\frac{s-1}{s}}(5,9)
$$

with $s$ as in (4.26) and $\gamma, \delta_{1}, \delta_{2}$ as in (4.28). Note that (5.7), (5.8) and (5.9) hold for all $t>0$, in view of the continuity property of $u$ deduced above. Moreover, thanks to Beppo Levi's monotone convergence theorem, it is possible to compute the limit as $k \rightarrow+\infty$ in the integrals of equality (5.6) and hence obtain that, for any $\varphi \in C_{c}^{\infty}\left(B_{R} \times(0, T)\right)$ such that $\varphi(x, T)=0$ for any $x \in B_{R}$, the function $u_{h}^{R}$ satisfies

$$
\begin{aligned}
-\int_{0}^{T} \int_{B_{R}} u_{h}^{R} \varphi_{t} d \mu d t= & \int_{0}^{T} \int_{B_{R}}\left(u_{h}^{R}\right)^{m} \Delta \varphi d \mu d t+\int_{0}^{T} \int_{B_{R}}\left(u_{h}^{R}\right)^{p} \varphi d \mu d t \\
& +\int_{B_{R}} u_{0, h}(x) \varphi(x, 0) d \mu .
\end{aligned}
$$

Observe that all the integrals in (5.10) are finite, hence $u_{h}^{R}$ is a solution to problem (5.1), where we replace $T_{k}\left(u^{p}\right)$ with $u^{p}$ itself, in the sense of Definition 3.1. Indeed, we have, due to (5.7), $u_{h}^{R} \in L^{m}\left(B_{R} \times(0, T)\right)$ hence $u_{h}^{R} \in L^{1}\left(B_{R} \times(0, T)\right)$. Moreover, due to (5.8), $u_{h}^{R} \in L^{p}\left(B_{R} \times(0, T)\right)$ indeed we can write

$$
\begin{aligned}
\int_{0}^{T} \int_{B_{R}}\left(u_{h}^{R}\right)^{p} d \mu d t & =\int_{0}^{T}\left\|u_{h}^{R}\right\|_{L^{p}\left(B_{R}\right)}^{p} d t \\
& \leq \int_{0}^{T}\left(C t^{-\gamma_{p}}\left\|u_{0, h}\right\|_{L^{p_{0}\left(B_{R}\right)}}^{\delta_{p}}\right)^{p} d t \\
& =C^{p}\left\|u_{0, h}\right\|_{L^{p_{0}\left(B_{R}\right)}}^{p \delta_{p}} \int_{0}^{T} t^{-p \gamma_{p}} d t
\end{aligned}
$$

Now observe that the integral in (5.11) is finite if and only if $p \gamma_{p}<1$. The latter reads $p>m+\frac{2}{N}$, which is guaranteed by the hypotheses of Theorem 2.2.

Let us now observe that, for any $h>0$, the sequence of solutions $\left\{u_{h}^{R}\right\}_{R>0}$ is monotone increasing in $R$, hence it has a pointwise limit as $R \rightarrow+\infty$. We call its limit function $u_{h}$ so that

$$
u_{h}^{R} \longrightarrow u_{h} \text { as } R \rightarrow+\infty \text { pointwise. }
$$

In view of (5.2), (5.3), (5.4), (5.7), (5.8), (5.9), the right-hand side of (5.5) is independent of $k$ and $R$. So, $\left(u_{h}\right)^{\frac{m+1}{2}} \in H^{1}\left((\tau, T) ; L^{2}(M)\right)$. Therefore, $\left(u_{h}\right)^{\frac{m+1}{2}} \in$ $C\left([\tau, T] ; L^{2}(M)\right)$. Since $u_{0} \in L^{m}(M) \cap L^{p_{0}}(M)$, there exists $k_{0}>0$ and $k_{1}>0$ such that

$$
\begin{aligned}
& \left\|u_{0 h}\right\|_{L^{m}\left(B_{R}\right)} \leq k_{0} \quad \forall h>0, \quad \forall R>0, \\
& \left\|u_{0 h}\right\|_{L^{p_{0}\left(B_{R}\right)} \leq k_{1}} \quad \forall h>0, \quad \forall R>0 .
\end{aligned}
$$


Note that, in view of (5.12), the norms in (5.7), (5.8) and (5.9) do not depend on $R$ (see Lemma 4.1, Proposition 4.2, Proposition 4.4 and Remark 4.5). Therefore, we pass to the limit as $R \rightarrow+\infty$ in (5.7), (5.8) and (5.9). By Fatou's Lemma,

$$
\begin{aligned}
\left\|u_{h}(t)\right\|_{L^{m}(M)} & \leq\left\|u_{0, h}\right\|_{L^{m}(M)}, \\
\left\|u_{h}(t)\right\|_{L^{p}(M)} & \leq C t^{-\gamma_{p}}\left\|u_{0, h}\right\|_{L^{p_{0}(M)}}^{\delta_{p}},
\end{aligned}
$$

furthermore, since $u_{h}^{R} \longrightarrow u_{h}$ as $R \rightarrow+\infty$ pointwise,

$$
\left\|u_{h}\right\|_{L^{\infty}(M)} \leq \Gamma t^{-\frac{\gamma}{m s}}\left\{\left\|u_{0, h}\right\|_{L^{p_{0}(M)}}^{\delta_{1}}+\frac{1}{m-1}\left\|u_{0, h}\right\|_{L^{p_{0}(M)}}^{\delta_{2}}\right\}^{\frac{1}{m s}}\left\|u_{0, h}\right\|_{L^{m}(M)}^{\frac{s-1}{s}},
$$

with $s$ as in (4.26) and $\gamma, \delta_{1}, \delta_{2}$ as in (4.28). Note that (5.13), (5.14) and (5.15) hold for all $t>0$, in view of the continuity property of $u_{h}^{R}$ deduced above.

Moreover, again by monotone convergence, it is possible to compute the limit as $R \rightarrow+\infty$ in the integrals of equality (5.10) and hence obtain that, for any $\varphi \in$ $C_{c}^{\infty}(M \times(0, T))$ such that $\varphi(x, T)=0$ for any $x \in M$, the function $u_{h}$ satisfies,

$$
\begin{aligned}
-\int_{0}^{T} \int_{M} u_{h} \varphi_{t} d \mu d t= & \int_{0}^{T} \int_{M}\left(u_{h}\right)^{m} \Delta \varphi d \mu d t+\int_{0}^{T} \int_{M}\left(u_{h}\right)^{p} \varphi d \mu d t \\
& +\int_{M} u_{0, h}(x) \varphi(x, 0) d \mu .
\end{aligned}
$$

Observe that, arguing as above, due to inequalities (5.13) and (5.14), all the integrals in (5.16) are well posed hence $u_{h}$ is a solution to problem (1.1), where we replace $u_{0}$ with $u_{0, h}$, in the sense of Definition 2.1. Finally, let us observe that $\left\{u_{0, h}\right\}_{h \geq 0}$ has been chosen in such a way that

$$
u_{0, h} \longrightarrow u_{0} \text { in } L^{m}(M) \cap L^{p_{0}}(M) .
$$

Observe also that $\left\{u_{h}\right\}_{h \geq 0}$ is a monotone increasing function in $h$ hence it has a limit as $h \rightarrow+\infty$. We call $u$ the limit function. In view (5.2), (5.3), (5.4), (5.7), (5.8), (5.9), (5.13), (5.14) and (5.15) the right-hand side of (5.5) is independent of $k, R$ and $h$. So, $u^{\frac{m+1}{2}} \in H^{1}\left((\tau, T) ; L^{2}(M)\right)$. Therefore, $u^{\frac{m+1}{2}} \in C\left([\tau, T] ; L^{2}(M)\right)$. Hence, we can pass to the limit as $h \rightarrow+\infty$ in (5.13), (5.14) and (5.15) and similarly to what we have seen above, we get

$$
\begin{aligned}
\|u(t)\|_{L^{m}(M)} & \leq\left\|u_{0}\right\|_{L^{m}(M)}, \\
\|u(t)\|_{L^{p}(M)} & \leq C t^{-\gamma_{p}}\left\|u_{0}\right\|_{L^{p_{0}(M)}}^{\delta_{p}},
\end{aligned}
$$

and

$$
\|u\|_{L^{\infty}(M)} \leq \Gamma t^{-\frac{\gamma}{m s}}\left\{\left\|u_{0}\right\|_{L^{p_{0}(M)}}^{\delta_{1}}+\frac{1}{m-1}\left\|u_{0}\right\|_{L^{p_{0}(M)}}^{\delta_{2}}\right\}^{\frac{1}{m s}}\left\|u_{0}\right\|_{L^{m}(M)}^{\frac{s-1}{s}},
$$


with $s$ as in (4.26) and $\gamma, \delta_{1}, \delta_{2}$ as in (4.28). Note that both (5.17), (5.18) and (5.19) hold for all $t>0$, in view of the continuity property of $u$ deduced above.

Moreover, again by monotone convergence, it is possible to compute the limit as $h \rightarrow+\infty$ in the integrals of equality (5.16) and hence obtain that, for any $\varphi \in$ $C_{c}^{\infty}(M \times(0, T))$ such that $\varphi(x, T)=0$ for any $x \in M$, the function $u$ satisfies,

$$
\begin{aligned}
-\int_{0}^{T} \int_{M} u \varphi_{t} d \mu d t= & \int_{0}^{T} \int_{M} u^{m} \Delta \varphi d \mu d t+\int_{0}^{T} \int_{M} u^{p} \varphi d \mu d t \\
& +\int_{M} u_{0}(x) \varphi(x, 0) d \mu .
\end{aligned}
$$

Observe that, due to inequalities (5.17) and (5.18), all the integrals in (5.20) are finite, hence $u$ is a solution to problem (1.1) in the sense of Definition 2.1.

Finally, let us discuss (2.6) and (2.4). Let $p_{0} \leq q<\infty$, and observe that, thanks to hypotheses $(c)$ and $(d), u_{0 h}$ satisfies hypothesis (2.3) for such $q$ and $q_{0}=p_{0}$ as $u_{0}$, then we have

$$
\left\|u_{h, k}^{R}(t)\right\|_{L^{q}\left(B_{R}\right)} \leq C t^{-\gamma_{q}}\left\|u_{0, h}\right\|_{L^{p_{0}\left(B_{R}\right)}}^{\delta_{q}} .
$$

Hence, due to (5.21), letting $k \rightarrow+\infty, R \rightarrow+\infty, h \rightarrow+\infty$, by Fatou's Lemma we deduce (2.4).

Now let $1<q<\infty$. If $u_{0} \in L^{q}(M) \cap L^{m}(M) \cap L^{p_{0}}(M)$, we choose the sequence $u_{0 h}$ in such a way that it further satisfies

$$
u_{0, h} \longrightarrow u_{0} \quad \text { in } L^{q}(M) \quad \text { as } h \rightarrow+\infty,
$$

and observe that $u_{0 h}$ satisfies also (2.5) for such $q$. Then, we have that

$$
\left\|u_{h, k}^{R}(t)\right\|_{L^{q}\left(B_{R}\right)} \leq\left\|u_{0, h}\right\|_{L^{q}\left(B_{R}\right)} .
$$

Hence, due to (5.22), letting $k \rightarrow+\infty, R \rightarrow+\infty, h \rightarrow+\infty$, by Fatou's Lemma we deduce (2.6).

\section{Estimates for $p>m$}

Lemma 6.1. Let $m>1, p>m$. Assume that inequalities (1.3) and (1.2) hold. Suppose that $u_{0} \in L^{\infty}\left(B_{R}\right), u_{0} \geq 0$. Let $1<q<\infty$ and assume that

$$
\left\|u_{0}\right\|_{L^{p \frac{N}{2}}\left(B_{R}\right)}<\tilde{\varepsilon}_{1}
$$

for a suitable $\tilde{\varepsilon}_{1}=\tilde{\varepsilon}_{1}\left(p, m, N, C_{p}, C_{s}, q\right)$ sufficiently small. Let $u$ be the solution of problem (3.2) in the sense of Definition 3.1, such that in addition $u \in C\left([0, T) ; L^{q}\left(B_{R}\right)\right)$. Then,

$$
\|u(t)\|_{L^{q}\left(B_{R}\right)} \leq\left\|u_{0}\right\|_{L^{q}\left(B_{R}\right)} \text { for all } t>0 .
$$


Proof. Since $u_{0}$ is bounded and $T_{k}$ is a bounded and Lipschitz function, by standard results, there exists a unique solution of problem (3.2) in the sense of Definition 3.1. We now multiply both sides of the differential equation in problem (3.2) by $u^{q-1}$, therefore

$$
\int_{B_{R}} u_{t} u^{q-1} d \mu=\int_{B_{R}} \Delta\left(u^{m}\right) u^{q-1} d \mu+\int_{B_{R}} T_{k}\left(u^{p}\right) u^{q-1} d \mu .
$$

We integrate by parts. This can be justified by standard tools, by an approximation procedure. Using the fact that $T\left(u^{p}\right) \leq u^{p}$, we can write

$$
\begin{aligned}
\frac{1}{q} \frac{d}{d t} \int_{B_{R}} u^{q} d \mu & \leq-m(q-1) \int_{B_{R}} u^{m+q-3}|\nabla u|^{2} d \mu+\int_{B_{R}} u^{p} u^{q-1} d \mu \\
& \leq-\frac{4 m(q-1)}{(m+q-1)^{2}} \int_{B_{R}}\left|\nabla\left(u^{\frac{m+q-1}{2}}\right)\right|^{2} d \mu+\int_{B_{R}} u^{p+q-1} d \mu .
\end{aligned}
$$

Now we take $c_{1}>0, c_{2}>0$ such that $c_{1}+c_{2}=1$. Thus,

$$
\int_{B_{R}}\left|\nabla\left(u^{\frac{m+q-1}{2}}\right)\right|^{2} d \mu=c_{1}\left\|\nabla\left(u^{\frac{m+q-1}{2}}\right)\right\|_{L^{2}\left(B_{R}\right)}^{2}+c_{2}\left\|\nabla\left(u^{\frac{m+q-1}{2}}\right)\right\|_{L^{2}\left(B_{R}\right)}^{2} .
$$

Take any $\alpha \in(0,1)$. Thanks to (1.3), (6.4) becomes

$$
\begin{gathered}
\int_{B_{R}}\left|\nabla\left(u^{\frac{m+q-1}{2}}\right)\right|^{2} d \mu \geq c_{1} C_{p}^{2}\|u\|_{L^{m+q-1}\left(B_{R}\right)}^{m+q-1}+c_{2}\left\|\nabla\left(u^{\frac{m+q-1}{2}}\right)\right\|_{L^{2}\left(B_{R}\right)}^{2} \\
\geq c_{1} C_{p}^{2}\|u\|_{L^{m+q-1}\left(B_{R}\right)}^{m+q-1}+c_{2}\left\|\nabla\left(u^{\frac{m+q-1}{2}}\right)\right\|_{L^{2}\left(B_{R}\right)}^{2+2 \alpha-2 \alpha} \\
\geq c_{1} C_{p}^{2}\|u\|_{L^{m+q-1}\left(B_{R}\right)}^{m+q-1}+c_{2} C_{p}^{2 \alpha}\|u\|_{L^{m+q-1}\left(B_{R}\right)}^{\alpha(m+q-1)}\left\|\nabla\left(u^{\frac{m+q-1}{2}}\right)\right\|_{L^{2}\left(B_{R}\right)}^{2-2 \alpha}
\end{gathered}
$$

Moreover, using the interpolation inequality, Hölder inequality and (1.2), we have

$$
\begin{aligned}
& \int_{B_{R}} u^{p+q-1} d \mu,=\|u\|_{L^{p+q-1}}^{p+q-1} \\
& \quad \leq\|u\|_{L^{m+q-1}\left(B_{R}\right)}^{\theta(p+q-1)}\|u\|_{L^{p+m+q-1}\left(B_{R}\right)}^{(1-\theta)(p+q-1)} \\
& \quad \leq\|u\|_{L^{m+q-1}\left(B_{R}\right)}^{\theta(p+q-1)}\left[\begin{array}{c}
\|u\|_{L^{p \frac{N}{2}\left(B_{R}\right)}}^{(1-\theta) \frac{p}{p+m+q-1}}\|u\|_{L^{(m+q-1) \frac{N}{N-2}\left(B_{R}\right)}}^{(1-\theta) \frac{m+q-1}{p+m+q-1}}
\end{array}\right]^{p+q-1} \\
& \quad \leq\|u\|_{L^{m+q-1}\left(B_{R}\right)}^{\theta(p+q-1)}\|u\|_{L^{p \frac{N}{2}\left(B_{R}\right)}}^{(1-\theta) \frac{p(p+q-1)}{p+m+q-1}}\left(\frac{1}{C_{S}}\left\|\nabla\left(u^{\left.\frac{m+q-1}{2}\right)}\right)\right\|_{L^{2}\left(B_{R}\right)}\right)^{2(1-\theta) \frac{p+q-1}{p+m+q-1}}
\end{aligned}
$$

where $\theta:=\frac{m(m+q-1)}{p(p+q-1)}$. By plugging (6.5) and (6.6) into (6.3), we obtain 


$$
\begin{aligned}
& \frac{1}{q} \frac{d}{d t}\|u(t)\|_{L^{q}\left(B_{R}\right)}^{q} \leq-\frac{4 m(q-1)}{(m+q-1)^{2}} c_{1} C_{p}^{2}\|u(t)\|_{L^{m+q-1}\left(B_{R}\right)}^{m+q-1} \\
& -\frac{4 m(q-1)}{(m+q-1)^{2}} c_{2} C_{p}^{2 \alpha}\|u(t)\|_{L^{m+q-1}\left(B_{R}\right)}^{\alpha(m+q-1)}\left\|\nabla\left(u^{\frac{m+q-1}{2}}\right)\right\|_{L^{2}\left(B_{R}\right)}^{2-2 \alpha} \\
& \quad+\tilde{C}\|u(t)\|_{L^{m+q-1}\left(B_{R}\right)}^{\theta(p+q-1)}\|u(t)\|_{L^{p \frac{N}{2}\left(B_{R}\right)}}^{(1-\theta) \frac{p(p+q-1)}{p+m+q-1}}\left\|\nabla\left(u^{\frac{m+q-1}{2}}\right)\right\|_{L^{2}\left(B_{R}\right)}^{2(1-\theta) \frac{p+q-1}{p+m+q-1}}
\end{aligned}
$$

where

$$
\tilde{C}=\left(\frac{1}{C_{S}}\right)^{2(1-\theta) \frac{p+q-1}{p+m+q-1}} .
$$

Let us now fix $\alpha \in(0,1)$ such that

$$
2-2 \alpha=2(1-\theta)\left(\frac{p+q-1}{p+m+q-1}\right) .
$$

Hence, we have

$$
\alpha=\frac{m}{p} .
$$

By substituting (6.9) into (6.7), we obtain

$$
\begin{aligned}
& \frac{1}{q} \frac{d}{d t}\|u(t)\|_{L^{q}\left(B_{R}\right)}^{q} \leq-\frac{4 m(q-1)}{(m+q-1)^{2}} c_{1} C_{p}^{2}\|u(t)\|_{L^{m+q-1}\left(B_{R}\right)}^{m+q-1} \\
& -\frac{1}{\tilde{C}}\left\{\frac{4 m(q-1) C}{(m+q-1)^{2}}-\|u(t)\| \frac{p(p+q-1)-m(m+q-1)}{p+m+q-1}{ }_{L^{p \frac{N}{2}}\left(B_{R}\right)}\right\} \\
& \times\|u(t)\|_{L^{m+q-1}\left(B_{R}\right)}^{\alpha(m+q-1)}\left\|\nabla\left(u^{\frac{m+q-1}{2}}\right)\right\|_{L^{2}\left(B_{R}\right)}^{2-2 \alpha},
\end{aligned}
$$

where $C$ has been defined in Remark 2.6. Observe that, thanks to hypothesis (6.1) and the continuity of the solution $u(t)$, there exists $t_{0}>0$ such that

$$
\|u(t)\|_{L^{p \frac{N}{2}}\left(B_{R}\right)} \leq 2 \tilde{\varepsilon}_{1} \text { for any } t \in\left(0, t_{0}\right] .
$$

Hence, (6.10) becomes, for any $t \in\left(0, t_{0}\right]$

$$
\begin{aligned}
\frac{1}{q} & \frac{d}{d t}\|u(t)\|_{L^{q}\left(B_{R}\right)}^{q} \leq-\frac{4 m(q-1)}{(m+q-1)^{2}} c_{1} C_{p}^{2}\|u(t)\|_{L^{m+q-1}\left(B_{R}\right)}^{m+q-1} \\
& -\frac{1}{\tilde{C}}\left\{\frac{4 m(q-1) C}{(m+q-1)^{2}}-2 \tilde{\varepsilon}_{1}^{\frac{p(p+q-1)-m(m+q-1)}{p+m+q-1}}\right\}\|u(t)\|_{L^{m+q-1}\left(B_{R}\right)}^{\alpha(m+q-1)}\left\|\nabla\left(u^{\frac{m+q-1}{2}}\right)\right\|_{L^{2}\left(B_{R}\right)}^{2-2 \alpha} \\
& \leq 0,
\end{aligned}
$$


provided $\tilde{\varepsilon}_{1}$ is small enough. Hence, we have proved that $\|u(t)\|_{L^{q}\left(B_{R}\right)}$ is decreasing in time for any $t \in\left(0, t_{0}\right]$, i.e.

$$
\|u(t)\|_{L^{q}\left(B_{R}\right)} \leq\left\|u_{0}\right\|_{L^{q}\left(B_{R}\right)} \text { for any } t \in\left(0, t_{0}\right] .
$$

In particular, inequality (6.11) holds $q=p \frac{N}{2}$. Hence, we have

$$
\|u(t)\|_{L^{p \frac{N}{2}\left(B_{R}\right)}} \leq\left\|u_{0}\right\|_{L^{p \frac{N}{2}}\left(B_{R}\right)}<\tilde{\varepsilon}_{1} \text { for any } t \in\left(0, t_{0}\right] .
$$

Now, we can repeat the same argument in the time interval $\left(t_{0}, t_{1}\right]$ where $t_{1}$ is chosen, thanks to the continuity of $u(t)$, in such a way that

$$
\|u(t)\| \leq 2 \tilde{\varepsilon}_{1} \text { for any } t \in\left(t_{0}, t_{1}\right] .
$$

Thus, we get

$$
\|u(t)\|_{L^{q}\left(B_{R}\right)} \leq\left\|u_{0}\right\|_{L^{q}\left(B_{R}\right)} \text { for any } t \in\left(0, t_{1}\right] .
$$

Iterating this procedure we obtain the thesis.

Proposition 6.2. Let $m>1, p>m, R>0, u_{0} \in L^{\infty}\left(B_{R}\right), u_{0} \geq 0$. Let

$$
r>\frac{N}{2}, \quad s=1+\frac{2}{N}-\frac{1}{r} .
$$

Suppose that (2.11) holds for $\varepsilon_{1}=\varepsilon_{1}\left(p, m, N, r, C_{s}, C_{p}\right)$ sufficiently small. Let $u$ be the solution to problem (3.2), such that in addition $u \in C\left([0, T) ; L^{q}\left(B_{R}\right)\right)$ for any $1<q<+\infty$ and $T>0$. Let $M$ support the Sobolev and Poincaré inequalities (1.2) and (1.3). Then, there exists $\Gamma=\Gamma\left(N, m, l, C_{s}\right)>0$ independent of $T$ such that, for all $t>0$,

$$
\|u(t)\|_{L^{\infty}\left(B_{R}\right)} \leq \Gamma\left\|u_{0}\right\|_{L^{m}\left(B_{R}\right)}^{\frac{s-1}{s}}\left[\left\|u_{0}\right\|_{L^{p r}\left(B_{R}\right)}^{p}+\frac{1}{(m-1) t}\left\|u_{0}\right\|_{L^{r}\left(B_{R}\right)}\right]^{\frac{1}{m s}} .
$$

Remark 6.3. If in Proposition 6.2, in addition, we assume that for some $k_{0}>0$

$$
\max \left\{\left\|u_{0}\right\|_{L^{m}\left(B_{R}\right)} ;\left\|u_{0}\right\|_{L^{p r}\left(B_{R}\right)} ;\left\|u_{0}\right\|_{L^{r}\left(B_{R}\right)}\right\} \leq k_{0} \text { for every } R>0,
$$

then the bound from above for $\|u(t)\|_{L^{\infty}\left(B_{R}\right)}$ in (6.13) is independent of $R$.

Proof of Proposition 6.2. Let us set $w=u(\cdot, t)$. Observe that $w^{m} \in H_{0}^{1}\left(B_{R}\right)$ and $w \geq 0$. Due to Proposition 3.6 we know that

$$
-\Delta\left(w^{m}\right) \leq\left[w^{p}+\frac{w}{(m-1) t}\right] .
$$

Observe that, since $u_{0} \in L^{\infty}\left(B_{R}\right)$ also $w \in L^{\infty}\left(B_{R}\right)$. Due to (6.12), we can apply Proposition 3.4, so we have that

$$
\|w\|_{L^{\infty}\left(B_{R}\right)}^{m} \leq \frac{s}{s-1}\left(\frac{1}{C_{S}}\right)^{\frac{2}{s}}\left\|w^{p}+\frac{w}{(m-1) t}\right\|_{L^{r}\left(B_{R}\right)}^{\frac{1}{s}}\left\|w^{m}\right\|_{L^{1}\left(B_{R}\right)}^{\frac{s-1}{s}} .
$$


Therefore

$$
\|w\|_{L^{\infty}\left(B_{R}\right)}^{m} \leq \frac{s}{s-1}\left(\frac{1}{C_{s}}\right)^{\frac{2}{s}}\left\{\left\|w^{p}\right\|_{L^{r}\left(B_{R}\right)}+\frac{1}{(m-1) t}\|w\|_{L^{r}\left(B_{R}\right)}\right\}^{\frac{1}{s}}\|w\|_{L^{m}\left(B_{R}\right)}^{m \frac{s-1}{s}},
$$

where $s$ has been defined in (6.12). In view of (2.11) with a suitable $\varepsilon_{1}$, since $u_{0} \in$ $L^{\infty}\left(B_{R}\right)$, we can apply Lemma 6.1. Hence, we obtain

$$
\left\|w^{p}\right\|_{L^{r}\left(B_{R}\right)}=\|w\|_{L^{p r}\left(B_{R}\right)}^{p} \leq\left\|u_{0}\right\|_{L^{p r}\left(B_{R}\right)}^{p} .
$$

Similarly, again for an appropriate $\varepsilon_{1}$ in (2.11), since $u_{0} \in L^{\infty}\left(B_{R}\right)$, we can apply Lemma 6.1 and obtain

$$
\|w\|_{L^{r}\left(B_{R}\right)} \leq\left\|u_{0}\right\|_{L^{r}\left(B_{R}\right)} .
$$

Plugging (6.15) and (6.16) into (6.14), we obtain

$$
\begin{aligned}
\|w\|_{L^{\infty}\left(B_{R}\right)}^{m} & \leq \frac{s}{s-1}\left(\frac{1}{C_{s}}\right)^{\frac{2}{s}}\left\{\|w\|_{L^{p r}\left(B_{R}\right)}^{p}+\frac{1}{(m-1) t}\|w\|_{L^{r}\left(B_{R}\right)}\right\}^{\frac{1}{s}}\|w\|_{L^{m}\left(B_{R}\right)}^{m \frac{s-1}{s}} \\
& \leq \frac{s}{s-1}\left(\frac{1}{C_{S}}\right)^{\frac{2}{s}}\left\{\left\|u_{0}\right\|_{L^{p r}\left(B_{R}\right)}^{p}+\frac{1}{(m-1) t}\left\|u_{0}\right\|_{L^{r}\left(B_{R}\right)}\right\}^{\frac{1}{s}}\|w\|_{L^{m}\left(B_{R}\right)}^{m} .
\end{aligned}
$$

Moreover, since $u_{0} \in L^{\infty}\left(B_{R}\right)$, we can apply Lemma 6.1 to $w$ with $q=m$. Thus, from (6.2) with $q=m$ we get

$$
\|w\|_{L^{\infty}\left(B_{R}\right)} \leq\left[\frac{s}{s-1}\left(\frac{1}{C_{s}}\right)^{\frac{2}{s}}\right]^{\frac{1}{m}}\left\|u_{0}\right\|_{L^{m}\left(B_{R}\right)}^{\frac{s-1}{s}}\left[\left\|u_{0}\right\|_{L^{p r}\left(B_{R}\right)}^{p}+\frac{1}{(m-1) t}\left\|u_{0}\right\|_{L^{r}\left(B_{R}\right)}\right]^{\frac{1}{m s}}
$$

We define

$$
\Gamma:=\left[\frac{s}{s-1}\left(\frac{1}{C_{s}}\right)^{\frac{2}{s}}\right]^{\frac{1}{m}}
$$

Then, from (6.17) we get

$$
\|w\|_{L^{\infty}\left(B_{R}\right)} \leq \Gamma\left\|u_{0}\right\|_{L^{m}\left(B_{R}\right)}^{\frac{s-1}{s}}\left[\left\|u_{0}\right\|_{L^{p r}\left(B_{R}\right)}^{p}+\frac{1}{(m-1) t}\left\|u_{0}\right\|_{L^{r}\left(B_{R}\right)}\right]^{\frac{1}{m s}} .
$$

Proof. The proof of Theorem 2.5 follows the same line of arguments of that of Theorem 2.2, with minor differences. Let $\left\{u_{0, h}\right\}_{h \geq 0}$ be a family of functions such that

(a) $u_{0, h} \in L^{\infty}(M) \cap C_{c}^{\infty}(M)$ for all $h \geq 0$,

(b) $u_{0, h} \geq 0$ for all $h \geq 0$,

(c) $u_{0, h_{1}} \leq u_{0, h_{2}}$ for any $h_{1}<h_{2}$,

(d) $u_{0, h} \longrightarrow u_{0}$ in $L^{\theta}(M) \cap L^{p r}(M)$ where $\theta:=\min \{m, r\} \quad$ as $h \rightarrow+\infty$, 
Observe that, due to assumptions $(c)$ and $(d), u_{0, h}$ satisfies (2.11) for an appropriate $\varepsilon_{1}$ sufficiently small. Moreover, thanks by interpolation, since $m<p<p r$, we have

$$
u_{0, h} \longrightarrow u_{0} \text { in } L^{p}(M) \quad \text { as } h \rightarrow+\infty
$$

For any $R>0, k>0, h>0$, consider the problem

$$
\begin{cases}u_{t}=\Delta u^{m}+T_{k}\left(u^{p}\right) & \text { in } B_{R} \times(0,+\infty) \\ u=0 & \text { in } \partial B_{R} \times(0, \infty) \\ u=u_{0, h} & \text { in } B_{R} \times\{0\} .\end{cases}
$$

From standard results it follows that problem (6.19) has a solution $u_{h, k}^{R}$ in the sense of Definition 3.1; moreover, $u_{h, k}^{R} \in C\left([0, T] ; L^{q}\left(B_{R}\right)\right)$ for any $q>1$. Hence, it satisfies the inequalities in Lemma 6.1 and in Proposition 6.2, i.e. for any $t \in(0,+\infty)$,

$$
\begin{aligned}
& \left\|u_{h, k}^{R}(t)\right\|_{L^{m}\left(B_{R}\right)} \leq\left\|u_{0, h}\right\|_{L^{m}\left(B_{R}\right)} \\
& \left\|u_{h, k}^{R}(t)\right\|_{L^{p}\left(B_{R}\right)} \leq\left\|u_{0, h}\right\|_{L^{p}\left(B_{R}\right)} \\
& \left\|u_{h, k}^{R}\right\|_{L^{\infty}\left(B_{R}\right)} \leq \Gamma\left\|u_{0, h}\right\|_{L^{m}\left(B_{R}\right)}^{\frac{s-1}{s}}\left[\left\|u_{0, h}\right\|_{L^{p r}\left(B_{R}\right)}^{p}+\frac{1}{(m-1) t}\left\|u_{0, h}\right\|_{L^{r}\left(B_{R}\right)}\right]^{\frac{1}{m s}},
\end{aligned}
$$

with $r$ and $s$ as in (6.12) and $\Gamma$ as in (6.18). Arguing as in the proof of Theorem (2.6), we can pass to the limit as $k \rightarrow+\infty, R \rightarrow+\infty, h \rightarrow \infty$ obtaining a function $u$, which satisfies

$$
\begin{aligned}
\|u(t)\|_{L^{m}(M)} & \leq\left\|u_{0}\right\|_{L^{m}(M)}, \\
\|u(t)\|_{L^{p}(M)} & \leq\left\|u_{0}\right\|_{L^{p}(M)},
\end{aligned}
$$

and

$$
\|u\|_{L^{\infty}(M)} \leq \Gamma\left\|u_{0}\right\|_{L^{m}(M)}^{\frac{s-1}{s}}\left[\left\|u_{0}\right\|_{L^{p r}(M)}^{p}+\frac{1}{(m-1) t}\left\|u_{0}\right\|_{L^{r}(M)}\right]^{\frac{1}{m s}},
$$

with $r$ and $s$ as in (6.12) and $\Gamma$ as in (6.18). Moreover, for any $\varphi \in C_{c}^{\infty}(M \times(0, T))$ such that $\varphi(x, T)=0$ for any $x \in M$, the function $u$ satisfies

$$
\begin{aligned}
-\int_{0}^{T} \int_{M} u \varphi_{t} d \mu d t= & \int_{0}^{T} \int_{M} u^{m} \Delta \varphi d \mu d t+\int_{0}^{T} \int_{M} u^{p} \varphi d \mu d t \\
& +\int_{M} u_{0}(x) \varphi(x, 0) d \mu .
\end{aligned}
$$

Observe that, due to inequalities (6.20), (6.21) and (6.22), all the integrals in (6.23) are finite, hence $u$ is a solution to problem (1.1) in the sense of Definition 2.1. Finally, using hypothesis (2.12), inequality (2.13) can be derived exactly as (2.6). 


\section{Proofs of Theorems 2.8 and 2.9}

We use the following Aronson-Benilan-type estimate (see [2]; see also [37, Proposition 2.3]); it can be shown exactly as Proposition 3.6.

Proposition 7.1. Let $m>1, p>m, u_{0} \in H_{0}^{1}\left(B_{R}\right) \cap L^{\infty}\left(B_{R}\right), u_{0} \geq 0$. Let $u$ be the solution to problem (7.1). Then, for a.e. $t \in(0, T)$,

$$
-\Delta u^{m}(\cdot, t) \leq \rho u^{p}(\cdot, t)+\frac{\rho}{(m-1) t} u(\cdot, t) \text { in } \mathfrak{D}^{\prime}\left(B_{R}\right) .
$$

For any $R>0$, consider the following approximate problem

$$
\begin{cases}\rho(x) u_{t}=\Delta u^{m}+\rho(x) u^{p} & \text { in } B_{R} \times(0, T) \\ u=0 & \text { in } \partial B_{R} \times(0, T) \\ u=u_{0} & \text { in } B_{R} \times\{0\},\end{cases}
$$

where $B_{R}$ denotes the Euclidean ball with radius $R$ and centre in the origin $O$.

We exploit the following estimate, which can be proved as that in Lemma 4.1.

Lemma 7.2. Let

$$
m>1, \quad p>m+\frac{2}{N} .
$$

Suppose that inequality (1.5) holds. Suppose that $u_{0} \in L^{\infty}\left(B_{R}\right), u_{0} \geq 0$. Let $1<q<\infty, p_{0}$ be as in (2.1) and assume that

$$
\left\|u_{0}\right\|_{L_{\rho}^{p_{0}}\left(B_{R}\right)}<\bar{\varepsilon}
$$

for $\bar{\varepsilon}=\bar{\varepsilon}\left(p, m, C_{s}, q\right)$ small enough. Let u be the solution of problem (7.1), such that in addition $u \in C\left([0, T), L_{\rho}^{q}\left(B_{R}\right)\right)$ for any $q \in(1,+\infty)$, for any $T>0$. Then,

$$
\|u(t)\|_{L_{\rho}^{q}\left(B_{R}\right)} \leq\left\|u_{0}\right\|_{L_{\rho}^{q}\left(B_{R}\right)} \text { for all } t>0 .
$$

The following smoothing estimate is also used; the proof is the same as that of Proposition 4.2.

Proposition 7.3. Let

$$
m>1, \quad p>m+\frac{2}{N},
$$

Assume (2.16) and (1.5). Suppose that $u_{0} \in L^{\infty}\left(B_{R}\right), u_{0} \geq 0$. Let $u$ be the solution of problem (7.1), such that in addition $u \in C\left([0, T), L_{\rho}^{q}\left(B_{R}\right)\right)$ for any $q \in$ $(1,+\infty)$, for any $T>0$. Assume that (2.2) holds for $\varepsilon_{0}=\varepsilon_{0}\left(p, m, N, r, C_{s}\right)$ sufficiently small. There exists $C\left(m, q_{0}, C_{s}, \varepsilon, N, q\right)>0$ such that

$$
\|u(t)\|_{L_{\rho}^{q}\left(B_{R}\right)} \leq C t^{-\gamma_{q}}\left\|u_{0}\right\|_{L_{\rho}^{q_{0}\left(B_{R}\right)}}^{\delta_{q}} \quad \text { for all } t>0
$$


where

$$
\gamma_{q}=\left(\frac{1}{q_{0}}-\frac{1}{q}\right) \frac{N q_{0}}{2 q_{0}+N(m-1)} ; \quad \delta_{q}=\frac{q_{0}}{q}\left(\frac{q+\frac{N}{2}(m-1)}{q_{0}+\frac{N}{2}(m-1)}\right) .
$$

Proof of Theorem 2.8. The conclusion follows by repeating the same arguments as in the proof of Theorem 2.2. We use Lemma 7.2 instead of Lemma 4.1, Proposition 7.3 instead of 4.2 and Proposition 7.1 instead of Proposition 3.6.

\subsection{Proof of Theorem 2.9}

We consider problem (7.1). We use the following estimate, which can be proved as that in Lemma 6.1.

Lemma 7.4. Let

$$
m>1, \quad p>m \text {. }
$$

Assume that (1.5) and (1.6) hold. Suppose that $u_{0} \in L^{\infty}\left(B_{R}\right), u_{0} \geq 0$. Let $1<q<\infty$ and assume that and assume that

$$
\left\|u_{0}\right\|_{L^{p \frac{N}{2}}\left(B_{R}\right)}<\tilde{\varepsilon}_{1}
$$

for a suitable $\tilde{\varepsilon}_{1}=\tilde{\varepsilon}_{1}\left(p, m, N, C_{p}, C_{s}, q\right)$ sufficiently small. Let $u$ be the solution of problem (7.1), such that in addition $u \in C\left([0, T), L^{q}\left(B_{R}\right)\right)$ for any $q \in$ $(1,+\infty)$, for any $T>0$. Then,

$$
\|u(t)\|_{L^{q}\left(B_{R}\right)} \leq\left\|u_{0}\right\|_{L^{q}\left(B_{R}\right)} \text { for all } t>0 .
$$

Proof of Theorem 2.9. The conclusion follows arguing step by step as in the proof of Theorem 2.5. We use Lemma 7.4 instead of Lemma 6.1 and Proposition 7.1 instead of Proposition 3.6.

\section{Acknowledgements}

The first and third authors are partially supported by the PRIN project 201758MTR2 "Direct and inverse problems for partial differential equations: theoretical aspects and applications" (Italy). All authors are members of the Gruppo Nazionale per 1'Analisi Matematica, la Probabilità e le loro Applicazioni (GNAMPA) of the Istituto Nazionale di Alta Matematica (INdAM). The third author is partially supported by GNAMPA Projects 2019, 2020.

Funding Open access funding provided by Politecnico di Milano within the CRUICARE Agreement. 
Open Access. This article is licensed under a Creative Commons Attribution 4.0 International License, which permits use, sharing, adaptation, distribution and reproduction in any medium or format, as long as you give appropriate credit to the original author(s) and the source, provide a link to the Creative Commons licence, and indicate if changes were made. The images or other third party material in this article are included in the article's Creative Commons licence, unless indicated otherwise in a credit line to the material. If material is not included in the article's Creative Commons licence and your intended use is not permitted by statutory regulation or exceeds the permitted use, you will need to obtain permission directly from the copyright holder. To view a copy of this licence, visit http://creativecommons.org/licenses/ by/4.0/.

Publisher's Note Springer Nature remains neutral with regard to jurisdictional claims in published maps and institutional affiliations.

\section{REFERENCES}

[1] D. Alikakos, $L^{p}$ bounds of solutions of reaction-diffusion equations, Comm. Partial Differential Equations 4 (1979), 827-868 .

[2] D. G. Aronson, P. Bénilan, Regularité des solutions de l'éequation des milieux poreus dans $\mathbb{R}^{N}, \mathrm{C}$. R. Acad. Sci. Paris Ser. A-B 288 (1979), 103-105 .

[3] D. Aronson, M.G. Crandall, L.A. Peletier, Stabilization of solutions of a degenerate nonlinear diffusion problem, Nonlinear Anal. 6 (1982), 1001-1022.

[4] L. Boccardo, G. Croce, "Elliptic partial differential equations. Existence and regularity of distributional solutions", De Gruyter, Studies in Mathematics, 55, 2013.

[5] C. Bandle, M.A. Pozio, A. Tesei, The Fujita exponent for the Cauchy problem in the hyperbolic space, J. Differential Equations 251 (2011), 2143-2163.

[6] M. Bonforte, G. Grillo, Asymptotics of the porous media equations via Sobolev inequalities, J. Funct. Anal. 225 (2005), 33-62.

[7] X. Chen, M. Fila, J.S. Guo, Boundedness of global solutions of a supercritical parabolic equation, Nonlinear Anal. 68 (2008), 621-628.

[8] H. Fujita, On the blowing up of solutions of the Cauchy problem for $u_{t}=\Delta u+u^{1+\alpha}$, J. Fac. Sci. Univ. Tokyo Sect. I 13 (1966), 109-124.

[9] Y. Fujishima, K. Ishige, Blow-up set for type I blowing up solutions for a semilinear heat equation, Ann. Inst. H. Poincaré Anal. Non Linéaire 31 (2014), 231-247.

[10] V.A. Galaktionov, J.L. Vázquez, Continuation of blowup solutions of nonlinear heat equations in several dimensions, Comm. Pure Appl. Math. 50 (1997), 1-67.

[11] A. Grigor'yan, Analytic and geometric background of recurrence and non-explosion of the Brownian motion on Riemannian manifolds, Bull. Amer. Math. Soc. 36 (1999), 135-249.

[12] A. Grigor'yan, "Heat Kernel and Analysis on Manifolds", AMS/IP Studies in Advanced Mathematics, 47, American Mathematical Society, Providence, RI; International Press, Boston, MA, 2009.

[13] G. Grillo, K. Ishige, M. Muratori, Nonlinear characterizations of stochastic completeness, J. Math. Pures Appl. 139 (2020), 63-82.

[14] G.Grillo, G. Meglioli, F. Punzo, Smoothing effects and infinite time blowup for reaction-diffusion equations: an approach via Sobolev and Poincaré inequalities, J. Math. Pures Appl. (to appear)

[15] G. Grillo, M. Muratori, Radial fast diffusion on the hyperbolic space, Proc. Lond. Math. Soc. 109 (2014), 283-317.

[16] G. Grillo, M. Muratori, Smoothing effects for the porous medium equation on Cartan-Hadamard manifolds, Nonlinear Anal. 131 (2016), 346-362.

[17] G. Grillo, M. Muratori, M.M. Porzio, Porous media equations with two weights: smoothing and decay properties of energy solutions via Poincaré inequalities, Discrete Contin. Dyn. Syst. 33 (2013), 3599-3640.

[18] G. Grillo, M. Muratori, F. Punzo, The porous medium equation with large initial data on negatively curved Riemannian manifolds, J. Math. Pures Appl. 113 (2018), 195-226. 
[19] G. Grillo, M. Muratori, F. Punzo, The porous medium equation with measure data on negatively curved Riemannian manifolds, J. European Math. Soc. 20 (2018), 2769-2812.

[20] G. Grillo, M. Muratori, F. Punzo, Blow-up and global existence for the porous medium equation with reaction on a class of Cartan-Hadamard manifolds, J. Diff. Eq. 266 (2019), 4305-4336.

[21] G. Grillo, M. Muratori, J.L. Vázquez, The porous medium equation on Riemannian manifolds with negative curvature. The large-time behaviour, Adv. Math. 314 (2017), 328-377.

[22] K. Hayakawa, On nonexistence of global solutions of some semilinear parabolic differential equations, Proc. Japan Acad. 49 (1973), 503-505.

[23] S. Kamin, P. Rosenau, Nonlinear thermal evolution in an inhomogeneous medium, J. Math. Phys. 23 (1982), 1385-1390.

[24] D. Kinderlehrer, G. Stampacchia, "An Introduction to Variational Inequalities and Their Applications", Academic Press, New York, 1980.

[25] H.A. Levine, The role of critical exponents in blow-up theorems, SIAM Rev. 32 (1990), 262-288.

[26] A.V. Martynenko, A. F. Tedeev, On the behavior of solutions of the Cauchy problem for a degenerate parabolic equation with nonhomogeneous density and a source, (Russian) Zh. Vychisl. Mat. Mat. Fiz. 48 (2008), no. 7, 1214-1229; transl. in Comput. Math. Math. Phys. 48 (2008), no. 7, 1145-1160.

[27] A.V. Martynenko, A.F. Tedeev, V.N. Shramenko, The Cauchy problem for a degenerate parabolic equation with inhomogenous density and a source in the class of slowly vanishing initial functions (Russian) Izv. Ross. Akad. Nauk Ser. Mat. 76 (2012), no. 3, 139-156; transl. in Izv. Math. 76 (2012), no. 3, 563-580.

[28] A.V. Martynenko, A.F. Tedeev, V.N. Shramenko, On the behavior of solutions of the Cauchy problem for a degenerate parabolic equation with source in the case where the initial function slowly vanishes, Ukrainian Math. J. 64 (2013), 1698-1715.

[29] P. Mastrolia, D. D. Monticelli, F. Punzo, Nonexistence of solutions to parabolic differential inequalities with a potential on Riemannian manifolds, Math. Ann. 367 (2017), 929-963.

[30] G. Meglioli, F. Punzo, Blow-up and global existence for solutions to the porous medium equation with reaction and slowly decaying density, J. Diff. Eq., 269 (2020), 8918-8958.

[31] G. Meglioli, F. Punzo, Blow-up and global existence for solutions to the porous medium equation with reaction and fast decaying density, Nonlin. Anal. 203 (2021), 112187.

[32] N. Mizoguchi, F. Quirós, J.L. Vázquez, Multiple blow-up for a porous medium equation with reaction, Math. Ann. 350 (2011), 801-827.

[33] F. Punzo, Support properties of solutions to nonlinear parabolic equations with variable density in the hyperbolic space, Discrete Contin. Dyn. Syst. Ser. S 5 (2012), 657-670.

[34] F. Punzo, Blow-up of solutions to semilinear parabolic equations on Riemannian manifolds with negative sectional curvature, J. Math. Anal. Appl. 387 (2012), 815-827.

[35] P. Quittner, The decay of global solutions of a semilinear heat equation, Discrete Contin. Dyn. Syst. 21 (2008), 307-318.

[36] P. Souplet, Morrey spaces and classification of global solutions for a supercritical semilinear heat equation in $\mathbb{R}^{n}$, J. Funct. Anal. 272 (2017), 2005-2037.

[37] P.E. Sacks, Global behavior for a class of nonlinear evolution equations, SIAM J. Math Anal. 16 (1985), 233-250.

[38] A.A. Samarskii, V.A. Galaktionov, S.P. Kurdyumov, A.P. Mikhailov, "Blow-up in Quasilinear Parabolic Equations", De Gruyter Expositions in Mathematics, 19. Walter de Gruyter \& Co., Berlin, 1995.

[39] J.L. Vázquez, The problems of blow-up for nonlinear heat equations. Complete blow-up and avalanche formation, Atti Accad. Naz. Lincei Cl. Sci. Fis. Mat. Natur. Rend. Lincei Mat. Appl. 15 (2004), 281-300.

[40] J.L. Vázquez, "The Porous Medium Equation. Mathematical Theory", Oxford Mathematical Monographs. The Clarendon Press, Oxford University Press, Oxford, 2007.

[41] J.L. Vázquez, Fundamental solution and long time behavior of the porous medium equation in hyperbolic space, J. Math. Pures Appl. 104 (2015), 454-484.

[42] Z. Wang, J. Yin, A note on semilinear heat equation in hyperbolic space, J. Differential Equations 256 (2014), 1151-1156.

[43] Z. Wang, J. Yin, Asymptotic behaviour of the lifespan of solutions for a semilinear heat equation in hyperbolic space, Proc. Roy. Soc. Edinburgh Sect. A 146 (2016) 1091-1114. 
[44] F.B. Weissler, $L^{p}$-energy and blow-up for a semilinear heat equation, Proc. Sympos. Pure Math. 45 (1986), 545-551.

[45] E. Yanagida, Behavior of global solutions of the Fujita equation, Sugaku Expositions 26 (2013), 129-147.

[46] Q.S. Zhang, Blow-up results for nonlinear parabolic equations on manifolds, Duke Math. J. 97 (1999), 515-539.

Gabriele Grillo, Giulia Meglioli and Fabio Punzo

Dipartimento di Matematica

Politecnico di Milano

Piazza Leonardo da Vinci 32

20133 Milano

Italy

E-mail: gabriele.grillo@polimi.it

Giulia Meglioli

E-mail: giulia.meglioli@polimi.it

Fabio Punzo

E-mail:fabio.punzo@polimi.it

Accepted: 6 March 2021 